\title{
p-adic periods, p-adic L-functions and the p-adic uniformization of Shimura curves
}

\author{
Massimo Bertolini ${ }^{1}$ \\ Henri Darmon ${ }^{2}$
}

\section{Contents}

1 Introduction . . . . . . . . . . . . . . . . . . . . . . . . . . . . 1

2 Definite quaternion algebras and graphs . . . . . . . . . . . . . . . . 4

3 The $p$-adic $L$-function . . . . . . . . . . . . . . . . . . . . . . . . 8

4 The theory of $p$-adic uniformization of Shimura curves . . . . . . . . . 14

$5 p$-adic Shintani cycles and special values of complex $L$-functions . . . . . 17

$6 p$-adic Shintani cycles and derivatives of $p$-adic $L$-functions . . . . . . . 19

7 Proof of theorem 6.1 . . . . . . . . . . . . . . . . . . . . . . . . 22

References . . . . . . . . . . . . . . . . . . . . . . . 30

\section{Introduction}

Let $E / \mathbb{Q}$ be a modular elliptic curve of conductor $N$, and let $p$ be a prime of split multiplicative reduction for $E$. Write $\mathbb{C}_{p}$ for a fixed completion of an algebraic closure of $\mathbb{Q}_{p}$. Tate's theory of $p$-adic uniformization of elliptic curves yields a rigid-analytic, $\operatorname{Gal}\left(\mathbb{C}_{p} / \mathbb{Q}_{p}\right)$-equivariant uniformization of the $\mathbb{C}_{p}$-points of $E$

$$
0 \rightarrow q^{\mathbb{Z}} \rightarrow \mathbb{C}_{p}^{\times} \stackrel{\Phi_{\text {Tate }}}{\longrightarrow} E\left(\mathbb{C}_{p}\right) \rightarrow 0
$$

where $q \in p \mathbb{Z}_{p}$ is the $p$-adic period of $E$.

Mazur, Tate and Teitelbaum conjectured in [MTT] that the cyclotomic $p$-adic $L$-function of $E / \mathbb{Q}$ vanishes at the central point to order one greater than that of its classical counterpart. Furthermore, they proposed a formula for the leading coefficient of such a $p$-adic $L$-function. In the special case where the analytic rank of $E(\mathbb{Q})$ is zero, they predicted that the ratio of the special value of the first derivative of the cyclotomic $p$-adic $L$-function and the algebraic part of the special value of the complex $L$-function of $E / \mathbb{Q}$ is equal to the quantity

$$
\frac{\log _{p}(q)}{\operatorname{ord}_{p}(q)}
$$

\footnotetext{
${ }^{1}$ Partially supported by GNSAGA (C.N.R.); M.U.R.S.T., progetto nazionale "Geometria algebrica"; Human Capital and Mobility Programme of the European Community, under contract ERBCH RXCT940557.

${ }^{2}$ Partially supported by grants from FCAR, NSERC and by an Alfred P. Sloan research award.
} 
(where $\log _{p}$ is Iwasawa's cyclotomic logarithm), which is defined purely in terms of the $p$-adic uniformization of $E$. Greenberg and Stevens [GS] gave a proof of this special case. See also the work of Boichut [Boi] in the case of analytic rank one.

The article [BD1] formulates an analogue of the conjectures of [MTT] in which the cyclotomic $\mathbb{Z}_{p}$-extension of $\mathbb{Q}$ is replaced by the anticyclotomic $\mathbb{Z}_{p}$-extension of an imaginary quadratic field $K$. When $p$ is split in $K$ and the sign of the functional equation of $L(E / K, s)$ is +1 , this conjecture relates the first derivative of the anticyclotomic $p$-adic $L$-function of $E$ to the anticyclotomic logarithm of the $p$-adic period of $E$. The present paper supplies a proof of this conjecture. Our proof is based on the theory of $p$-adic uniformization of Shimura curves.

More precisely, assume that $K$ is an imaginary quadratic field with $(\operatorname{disc}(K), N)=1$ such that:

(i) $p$ is split in $K$;

(ii) $E$ is semistable at the rational primes which divide $N$ and are inert in $K$;

(iii) the number of these rational primes is odd.

The complex $L$-function $L(E / K, s)$ of $E$ over $K$ has a functional equation and an analytic continuation to the whole complex plane. Under our assumptions, the sign of the functional equation of $L(E / K, s)$ is +1 (cf. [GZ], p. 71), and hence $L(E / K, s)$ vanishes to even order at $s=1$.

Fix a positive integer $c$ prime to $N$, and let $\mathcal{O}$ be the order of $K$ of conductor $c$. Let $H_{n}$ be the ring class field of $K$ of conductor $c p^{n}$, with $n \geq 0$, and let $H_{\infty}$ be the union of the $H_{n}$. By class field theory, the Galois group $\operatorname{Gal}\left(H_{\infty} / H_{0}\right)$ is identified with $\mathcal{O}^{\times} \backslash\left(\mathcal{O}_{K} \otimes \mathbb{Z}_{p}\right)^{\times} / \mathbb{Z}_{p}^{\times} \simeq \mathbb{Z}_{p} \times \mathbb{Z} /((p-1) / u) \mathbb{Z}$, with $u:=\frac{1}{2} \# \mathcal{O}^{\times}$. Moreover, $\operatorname{Gal}\left(H_{0} / K\right)$ is identified with the Picard group $\operatorname{Pic}(\mathcal{O})$. Set

$$
\mathbf{G}_{n}:=\operatorname{Gal}\left(H_{n} / K\right), \quad \mathbf{G}_{\infty}:=\operatorname{Gal}\left(H_{\infty} / K\right)
$$

Thus, $\mathbf{G}_{\infty}$ is isomorphic to the product of $\mathbb{Z}_{p}$ by a finite abelian group. Choose a prime $\mathfrak{p}$ of $K$ above $p$. Identify $K_{\mathfrak{p}}$ with $\mathbb{Q}_{p}$, and let

$$
\operatorname{rec}_{p}: \mathbb{Q}_{p}^{\times} \rightarrow \mathbf{G}_{\infty}
$$

be the reciprocity map of local class field theory. Define the integral completed group ring of $\mathbf{G}_{\infty}$ to be

$$
\mathbb{Z} \llbracket \mathbf{G}_{\infty} \rrbracket:=\lim _{n} \mathbb{Z}\left[\mathbf{G}_{n}\right]
$$

where the inverse limit is taken with respect to the natural projections of group rings.

In section 3, we recall the construction explained in [BD1], section 2.7 of an element

$$
\mathcal{L}_{p}(E / K) \in \mathbb{Z} \llbracket \mathbf{G}_{\infty} \rrbracket
$$

attached to $\left(E, H_{\infty} / K\right)$, which interpolates the special values $L(E / K, \chi, 1)$ of $L(E / K, s)$ twisted by finite order characters of $\mathbf{G}_{\infty}$. The construction of this $p$-adic $L$-function is based on the ideas of Gross $[\mathrm{Gr}]$ and a generalization due to Daghigh [Dag]. We will show that $\mathcal{L}_{p}(E / K)$ belongs to the augmentation ideal $I$ of $\mathbb{Z} \llbracket \mathbf{G}_{\infty} \rrbracket$. Let $\mathcal{L}_{p}^{\prime}(E / K)$ be the natural image of $\mathcal{L}_{p}(E / K)$ in $I / I^{2}=\mathbf{G}_{\infty}$. The element $\mathcal{L}_{p}^{\prime}(E / K)$ should be viewed as the first derivative of $\mathcal{L}_{p}(E / K)$ at the central point. 
Let $f=\sum_{n \geq 1} a_{n} q^{n}$ be the newform attached to $E$, and let

$$
\Omega_{f}:=4 \pi^{2} \iint_{\mathcal{H} / \Gamma_{0}(N)}|f(\tau)|^{2} d \tau \wedge i d \bar{\tau}
$$

be the Petersson inner product of $f$ with itself. We assume that $E$ is the strong Weil curve for the Shimura curve parametrization defined in section 4 . Set $d:=\operatorname{disc}(\mathcal{O})$, and let $n_{f}$ be the positive integer defined later in this introduction, and specified further in section 2. Our main result (stated in a special case: see theorem 6.4 for the general statement) is the following.

\section{Theorem 1.1}

Suppose that $c=1$. The equality (up to sign)

$$
\mathcal{L}_{p}^{\prime}(E / K)=\frac{\operatorname{rec}_{p}(q)}{\operatorname{ord}_{p}(q)} \sqrt{L(E / K, 1) \Omega_{f}^{-1} \cdot d^{\frac{1}{2}} u^{2} n_{f}}
$$

holds in $I / I^{2} \otimes \mathbb{Q}$.

For the convenience of the reader, we now briefly sketch the strategy of the proof of theorem 1.1.

Write the conductor $N$ of $E$ as $p N^{+} N^{-}$, where $N^{+}$, resp. $N^{-}$is divisible only by primes which are split, resp. inert in $K$. Under our assumptions, $N^{-}$has an odd number of prime factors, and $p N^{-}$is squarefree. Denote by $B$ the definite quaternion algebra over $\mathbb{Q}$ of discriminant $N^{-}$, and fix an Eichler order $R$ of $B$ of level $N^{+} p$. Let $\Gamma$ be the subgroup of elements of $\mathbb{Q}_{p}^{\times} \backslash R\left[\frac{1}{p}\right]^{\times}$whose norm has even $p$-adic valuation, and set $\mathcal{N}:=\operatorname{Hom}(\Gamma, \mathbb{Z})$. The module $\mathcal{N}$ is a free abelian group, and is equipped with the action of a Hecke algebra $\mathbb{T}$ attached to modular forms of level $N$ which are new at $N^{-} p$. In section 2 , we will also define a canonical free quotient $\mathcal{N}_{\text {sp }}$ of $\mathcal{N}$, which is stable for the action of $\mathbb{T}$ and is such that the image of $\mathbb{T}$ in $\operatorname{End}\left(\mathcal{N}_{\mathrm{sp}}\right)$ corresponds to modular forms which are split multiplicative at $p$. Let $\pi_{f}$ be the idempotent of $\mathbb{T} \otimes \mathbb{Q}$ associated with $f$, and let $n_{f}$ be a positive integer such that $\eta_{f}:=n_{f} \pi_{f}$ belongs to $\mathbb{T}$. Denote by $\mathcal{N}^{f}$ the submodule of $\mathcal{N}$ on which $\mathbb{T}$ acts via the character

$$
\phi_{f}: \mathbb{T} \rightarrow \mathbb{Z}, \quad T_{n} \mapsto a_{n}
$$

defined by $f$. By the multiplicity-one theorem, the module $\mathcal{N}^{f}$ is isomorphic to $\mathbb{Z}$. The operator $\eta_{f}$ yields a map (denoted in the same way by an abuse of notation) $\eta_{f}: \mathcal{N} \rightarrow \mathcal{N}^{f}$, which factors through $\mathcal{N}_{\text {sp }}$. We will define an element $\mathcal{L}_{p}\left(\mathcal{N}_{\mathrm{sp}} / K\right) \in$ $\mathcal{N}_{\text {sp }} \otimes \mathbb{Z} \llbracket \mathbf{G}_{\infty} \rrbracket$, such that (up to sign)

$$
\left(\eta_{f} \otimes \mathrm{id}\right)\left(\mathcal{L}_{p}\left(\mathcal{N}_{\mathrm{sp}} / K\right)\right)=c_{p} \cdot \mathcal{L}_{p}(E / K)
$$

where $c_{p}:=\operatorname{ord}_{p}(q)$. Recall that the derivative $\mathcal{L}_{p}^{\prime}(E / K)$ of $\mathcal{L}_{p}(E / K)$ belongs to $\mathcal{N}^{f} \otimes \mathbf{G}_{\infty}=\mathbf{G}_{\infty}$.

On the other hand, the module $\mathcal{N}$ is related to the theory of $p$-adic uniformization of Shimura curves. Let $\mathcal{B}$ be the indefinite quaternion algebra of discriminant $p N^{-}$, and let $\mathcal{R}$ be an Eichler order of $\mathcal{B}$ of level $N^{+}$. Write $X$ for the Shimura curve 
over $\mathbb{Q}$ associated with $\mathcal{R}$ (see section 4 ), and $J$ for the jacobian of $X$. A theorem of Cerednik ([Cer]), combined with the theory of jacobians of Mumford curves $([\mathrm{GVdP}])$, yields a rigid-analytic uniformization

$$
0 \rightarrow \Lambda \rightarrow \mathcal{N} \otimes \mathbb{C}_{p}^{\times} \stackrel{\Phi}{\longrightarrow} J\left(\mathbb{C}_{p}\right) \rightarrow 0
$$

where $\Lambda$ is the lattice of $p$-adic periods of $J$. The Tate uniformization (1) is obtained from the sequence (2) by applying the operator $\eta_{f}$ to the Hecke modules $\mathcal{N} \otimes \mathbb{C}_{p}^{\times}$ and $J\left(\mathbb{C}_{p}\right)$ of $(2)$. In particular, the $p$-adic period $q$ of $E$ can be viewed as an element of the module $\mathcal{N}^{f} \otimes \mathbb{C}_{p}^{\times}$, and in fact one checks it belongs to $\mathcal{N}^{f} \otimes \mathbb{Q}_{p}^{\times}=\mathbb{Q}_{p}^{\times}$. An explicit calculation of $p$-adic periods, combined with a formula for $L(E / K, 1)$ given in [Gr] and [Dag], will prove theorem 1.1.

A similar strategy was used in [BD2], when $p$ is inert in $K$ and the sign of the functional equation of $L(E / K, s)$ is -1 , to obtain a $p$-adic analytic construction of a Heegner point in terms of the first derivative of an anticyclotomic $p$-adic $L$-function.

It is worth observing that an analogous strategy has not (yet) proven to work in the case of the cyclotomic $\mathbb{Z}_{p}$-extension of $\mathbb{Q}$. The difficulty is that of relating in a natural way the construction of the cyclotomic $p$-adic $L$-function, which is defined in terms of modular symbols, to the $p$-adic uniformization of Shimura curves. P. Schneider [Sch] has proposed the definition of a $p$-adic $L$-function based on the notion, which stems directly from the theory of $p$-adic uniformization, of rigid-analytic modular symbol. C. Klingenberg $[\mathrm{Kl}]$ has proven an exceptional zero formula similar to theorem 1.1 for this rigid-analytic $p$-adic $L$-function. However, the relation (if any) between Schneider's $p$-adic $L$-function and the cyclotomic $p$-adic $L$-function considered in [MTT] is at present mysterious.

The reader is also referred to Teitelbaum's paper [T], where the theory of $p$-adic uniformization of Shimura curves is used to formulate analogues of the conjectures of [MTT] for cyclotomic $p$-adic $L$-functions attached to modular forms of higher weight.

The proof by Greenberg and Stevens [GS] of the cyclotomic "exceptional zero" formula of [MTT] follows a completely different strategy from the one of this paper, and is based on Hida's theory of $p$-adic families of modular forms.

Finally, let us mention that Kato, Kurihara and Tsuji [KKT] have recently announced more general results on the conjectures of [MTT], which make use of an Euler System constructed by Kato from modular units in towers of modular function fields.

\section{Definite quaternion algebras and graphs}

Keep the notations and assumptions of the introduction. In particular, recall that $K$ is an imaginary quadratic field, and $B$ is a definite quaternion algebra of discriminant $N^{-}$. Given a rational prime $\ell$, and orders $O$ of $K$ and $S$ of $B$, set

$$
K_{\ell}:=K \otimes \mathbb{Z}_{\ell}, \quad B_{\ell}:=B \otimes \mathbb{Z}_{\ell}, \quad O_{\ell}:=O \otimes \mathbb{Z}_{\ell}, \quad S_{\ell}:=S \otimes \mathbb{Z}_{\ell} .
$$

Denote by $\hat{\mathbb{Z}}=\prod \mathbb{Z}_{\ell}$ the profinite completion of $\mathbb{Z}$. Set

$$
\hat{K}:=K \otimes \hat{\mathbb{Z}}, \quad \hat{B}:=B \otimes \hat{\mathbb{Z}}, \quad \hat{O}:=O \otimes \hat{\mathbb{Z}}=\prod O_{\ell}, \quad \hat{S}:=S \otimes \hat{\mathbb{Z}}=\prod S_{\ell} .
$$


Fix an Eichler order $R$ of $B$ of level $N^{+} p$. Equip $R$ with an orientation, i.e., a collection of algebra homomorphisms

$$
\begin{array}{cc}
\mathfrak{o}_{\ell}^{+}: R \rightarrow \mathbb{Z} / \ell^{n} \mathbb{Z}, & \ell^{n} \| N^{+} p, \\
\mathfrak{o}_{\ell}^{-}: R \rightarrow \mathbb{F}_{\ell^{2}}, & \ell \mid N^{-} .
\end{array}
$$

The group $\hat{B}^{\times}$acts transitively (on the right) on the set of Eichler orders of level $N^{+} p$ by the rule

$$
S * \hat{b}:=\left(\hat{b}^{-1} \hat{S} \hat{b}\right) \cap B .
$$

The orientation on $R$ induces an orientation on $R * \hat{b}$, and the stabilizer of the oriented order $R$ is equal to $\mathbb{Q}^{\times} \hat{R}^{\times}$. This sets up a bijection between the set of oriented Eichler orders of level $N^{+} p$ and the coset space $\mathbb{Q}^{\times} \hat{R}^{\times} \backslash \hat{B}^{\times}$. Likewise, there is a bijection between the set of oriented Eicher orders of level $N^{+} p$ modulo conjugation by $B^{\times}$and the double coset space

$$
\hat{R}^{\times} \backslash \hat{B}^{\times} / B^{\times}
$$

Set $\Gamma_{+}:=\mathbb{Q}_{p}^{\times} \backslash R\left[\frac{1}{p}\right]^{\times}$and, as in the introduction, let $\Gamma$ be the image in $\Gamma_{+}$of the elements in $R\left[\frac{1}{p}\right]^{\times}$whose reduced norm has even $p$-adic valuation.

\section{Lemma 2.1}

$\Gamma$ has index 2 in $\Gamma_{+}$.

Proof. See [BD2], lemma 1.5.

Let $\mathcal{T}$ be the Bruhat-Tits tree associated with the local algebra $B_{p}$. The set of vertices $\mathcal{V}(\mathcal{T})$ of $\mathcal{T}$ is equal to the set of maximal orders in $B_{p}$. The set $\overrightarrow{\mathcal{E}}(\mathcal{T})$ of oriented edges of $\mathcal{T}$ is equal to the set of oriented Eichler orders of level $p$ in $B_{p}$. Thus, $\overrightarrow{\mathcal{E}}(\mathcal{T})$ can be identified with the coset space $\mathbb{Q}_{p}^{\times} R_{p}^{\times} \backslash B_{p}^{\times}$, by mapping $b_{p} \in B_{p}^{\times}$ to $R_{p} * b_{p}=b_{p}^{-1} R_{p} b_{p}$. Similarly, if $\underline{R}_{p}$ is a maximal order in $B_{p}$ containing $R_{p}$, we will identify $\mathcal{V}(\mathcal{T})$ with the coset space $\mathbb{Q}_{p}^{\times} \underline{R}_{p}^{\times} \backslash B_{p}^{\times}$. Define the graphs

$$
\mathcal{G}:=\mathcal{T} / \Gamma, \quad \mathcal{G}_{+}:=\mathcal{T} / \Gamma_{+}
$$

By strong approximation ([Vi], p. 61), there is an identification

$$
\overrightarrow{\mathcal{E}}\left(\mathcal{G}_{+}\right)=\hat{R}^{\times} \backslash \hat{B}^{\times} / B^{\times}
$$

of the set of oriented edges of $\mathcal{G}_{+}$with the set of conjugacy classes of oriented Eichler orders of level $N^{+} p$.

Fixing a vertex $v_{0}$ of $\mathcal{T}$ gives rise to an orientation of $\mathcal{T}$ in the following way. A vertex of $\mathcal{T}$ is called even, resp. odd if it has even, resp. odd distance from $v_{0}$. The direction of an edge is said to be positive if it goes from the even to the odd vertex. Since $\Gamma$ sends even vertices to even ones, and odd vertices to odd ones, the orientation of $\mathcal{T}$ induces an orientation of $\mathcal{G}$. Define a map

$$
\kappa: \mathcal{E}(\mathcal{G}) \rightarrow \overrightarrow{\mathcal{E}}\left(\mathcal{G}_{+}\right)
$$


from the set of edges of $\mathcal{G}$ to the set of oriented edges of $\mathcal{G}_{+}$, by mapping an edge $\left\{v, v^{\prime}\right\}(\bmod \Gamma)$ of $\mathcal{G}$, where $v$ and $v^{\prime}$ are vertices of $\mathcal{T}$ and we assume that $v$ is even, to the oriented edge $\left(v, v^{\prime}\right)\left(\bmod \Gamma_{+}\right)$of $\mathcal{G}_{+}$.

\section{Lemma 2.2}

The map $\kappa$ is a bijection.

Proof. Suppose that $\left(v, v^{\prime}\right)\left(\bmod \Gamma_{+}\right)=\left(u, u^{\prime}\right)\left(\bmod \Gamma_{+}\right)$. Thus, there is $\gamma \in \Gamma_{+}$ such that $\gamma v=u$ and $\gamma v^{\prime}=u^{\prime}$. If $v$ and $u$ are both even, $\gamma$ must belong to $\Gamma$, and this proves the injectivity of $\kappa$. As for surjectivity, $\left(v, v^{\prime}\right)\left(\bmod \Gamma_{+}\right)$is the image by $\kappa$ of $\left\{v, v^{\prime}\right\}(\bmod \Gamma)$ if $v$ is even, and of $\left\{w v, w v^{\prime}\right\}(\bmod \Gamma)$, where $w$ is any element of $\Gamma_{+}-\Gamma$, if $v$ is odd.

Given two vertices $v$ and $v^{\prime}$ of $\mathcal{T}$, write $\operatorname{path}\left(v, v^{\prime}\right)$ for the natural image in $\mathbb{Z}[\mathcal{E}(\mathcal{G})]$ of the unique geodesic on $\mathcal{T}$ joining $v$ with $v^{\prime}$. For example, if $v$ and $v^{\prime}$ are even vertices joined by 4 consecutive edges $e_{1}, e_{2}, e_{3}, e_{4}$, by our convention for orienting the edges of $\mathcal{T}, \operatorname{path}\left(v, v^{\prime}\right)$ is the image in $\mathbb{Z}[\mathcal{E}(\mathcal{G})]$ of $e_{1}-e_{2}+e_{3}-e_{4}$.

There is a coboundary map

$$
\partial^{*}: \mathbb{Z}[\mathcal{V}(\mathcal{G})] \rightarrow \mathbb{Z}[\mathcal{E}(\mathcal{G})]
$$

which maps the image in $\mathcal{V}(\mathcal{G})$ of an odd, resp. even vertex $v$ of $\mathcal{T}$ to the image in $\mathbb{Z}[\mathcal{E}(\mathcal{G})]$ of the formal sum of the edges of $\mathcal{T}$ emanating from $v$, resp. the opposite of this sum. There is also a boundary map

$$
\partial_{*}: \mathbb{Z}[\mathcal{E}(\mathcal{G})] \rightarrow \mathbb{Z}[\mathcal{V}(\mathcal{G})]
$$

which maps an edge $e$ to the difference $v^{\prime}-v$ of its vertices, where $v$ is the even vertex and $v^{\prime}$ is the odd vertex of $e$. The integral homology, resp. the integral cohomology of the graph $\mathcal{G}$ is defined by $H_{1}(\mathcal{G}, \mathbb{Z})=\operatorname{ker}\left(\partial_{*}\right)$, resp. $H^{1}(\mathcal{G}, \mathbb{Z})=\operatorname{coker}\left(\partial^{*}\right)$.

Let

$$
\langle,\rangle: \mathbb{Z}[\mathcal{E}(\mathcal{G})] \times \mathbb{Z}[\mathcal{E}(\mathcal{G})] \rightarrow \mathbb{Z}
$$

be the pairing on $\mathbb{Z}[\mathcal{E}(\mathcal{G})]$ defined by the rule $\left\langle e_{i}, e_{j}\right\rangle:=\omega_{e_{i}} \delta_{i j}$, where the $e_{i}$ are the elements of the standard basis of $\mathbb{Z}[\mathcal{E}(\mathcal{G})]$ and $\omega_{e_{i}}$ is the order of the stabilizer in $\Gamma$ of a lift of $e_{i}$ to $\mathcal{T}$. Likewise, let

$$
\langle\langle,\rangle: \mathbb{Z}[\mathcal{V}(\mathcal{G})] \times \mathbb{Z}[\mathcal{V}(\mathcal{G})] \rightarrow \mathbb{Z}
$$

be the pairing on $\mathbb{Z}[\mathcal{V}(\mathcal{G})]$ defined by $\left\langle\left\langle v_{i}, v_{j}\right\rangle\right\rangle:=\omega_{v_{i}} \delta_{i j}$, where the $v_{i}$ are the elements of the standard basis of $\mathbb{Z}[\mathcal{V}(\mathcal{G})]$ and $\omega_{v_{i}}$ is the order of the stabilizer in $\Gamma$ of a lift of $v_{i}$ to $\mathcal{T}$.

We use the notation $\mathcal{M}$ to indicate the module $H^{1}(\mathcal{G}, \mathbb{Z})$. Let $\bar{\Gamma}$ be the maximal torsion-free abelian quotient of $\Gamma$. As in the introduction, write $\mathcal{N}$ for $\operatorname{Hom}(\bar{\Gamma}, \mathbb{Z})$. Given an element $\gamma \in \Gamma$, denote by $\bar{\gamma}$ the natural image of $\gamma$ in $\bar{\Gamma}$.

\section{Lemma 2.3}

(i) The map from $\bar{\Gamma}$ to $H_{1}(\mathcal{G}, \mathbb{Z})$ which sends $\bar{\gamma} \in \bar{\Gamma}$ to the cycle path $\left(v_{0}, \gamma v_{0}\right)$, where $v_{0}$ is any vertex of $\mathcal{G}$ and $\gamma$ is any lift of $\bar{\gamma}$ to $\Gamma$, is an isomorphism. 
(ii) The map from $\mathcal{M}$ to $\mathcal{N}$ which sends $m \in \mathcal{M}$ to the homomorphism

$$
\bar{\gamma} \mapsto\left\langle\operatorname{path}\left(v_{0}, \gamma v_{0}\right), m\right\rangle
$$

is injective and has finite cokernel.

Proof (sketch). Part (i) is proved in [Se]. Part (ii) follows from part (i), and from the fact that the maps $\partial^{*}$ and $\partial_{*}$ are adjoint with respect to the pairings defined above.

Write $\mathcal{M}_{\text {sp }}$ for the maximal torsion-free quotient of $\mathcal{M} /(w+1) \mathcal{M}$, with $w \in \Gamma_{+}-\Gamma$. By part (i) of lemma 2.3, the action of $w \in \Gamma_{+}-\Gamma$ on $H_{1}(\mathcal{G}, \mathbb{Z})$ induces an action of $w$ on $\mathcal{N}$. Write $\mathcal{N}_{\mathrm{sp}}$ for the maximal torsion-free quotient of $\mathcal{N} /(w+1) \mathcal{N}$. We have an induced map from $\mathcal{M}_{\mathrm{sp}}$ to $\mathcal{N}_{\mathrm{sp}}$, which is injective and has finite cokernel.

The module $\mathbb{Z}[\mathcal{E}(\mathcal{G})]$ is equipped with the natural action of an algebra $\tilde{\mathbb{T}}$ generated over $\mathbb{Z}$ by the Hecke correspondences $T_{\ell}$ for $\ell \nmid N$ and $U_{\ell}$ for $\ell \mid N$, coming from its double coset description: see [BD1], sec. 1.5 . The module $H_{1}(\mathcal{G}, \mathbb{Z})$ is stable under the action of $\tilde{\mathbb{T}}$. Hence, by part (i) of lemma 2.3 , the algebra $\tilde{\mathbb{T}}$ also acts on the modules $\mathcal{N}$ and $\mathcal{N}_{\mathrm{sp}}$. Let $\mathbb{T}$ and $\mathbb{T}_{\mathrm{sp}}$ denote the image of $\tilde{\mathbb{T}}$ in $\operatorname{End}(\mathcal{N})$ and $\operatorname{End}\left(\mathcal{N}_{\mathrm{sp}}\right)$, respectively. Thus, there are natural surjections $\tilde{\mathbb{T}} \rightarrow \mathbb{T} \rightarrow \mathbb{T}_{\mathrm{sp}}$. By an abuse of notation, we will denote by $T_{\ell}$ and $U_{\ell}$ also the natural images in $\mathbb{T}$ and $\mathbb{T}_{\mathrm{sp}}$ of $T_{\ell}$ and $U_{\ell}$.

The next proposition clarifies the relation between the modules $\mathcal{N}$ and $\mathcal{N}_{\text {sp }}$ and the theory of modular forms.

\section{Proposition 2.4}

Let $\phi$ be an algebra homomorphism from $\mathbb{T}$, resp. $\mathbb{T}_{\mathrm{sp}}$ to $\mathbb{C}$, and let $a_{n}:=\phi\left(T_{n}\right)$. Then, the $a_{n}$ are the Fourier coefficients of a normalized eigenform of level $N$, which is new at $N^{-} p$, resp. is new at $N^{-} p$ and is split multiplicative at $p$. Conversely, any such modular form arises as above from a character of $\mathbb{T}$, resp. $\mathbb{T}_{\mathrm{sp}}$.

Proof. Eichler's trace formula identifies the Hecke-module $\mathbb{Z}[\mathcal{E}(\mathcal{G})]$ with a space of modular forms of level $N$ which are new at $N^{-}$. Moreover, the algebra $\mathbb{T}$ can also be viewed as the Hecke algebra of the module $\mathcal{M}$ defined above, and proposition 1.4 of [BD2] shows that $\mathcal{M}$ is equal to the " $p$-new" quotient of $\mathbb{Z}[\mathcal{E}(\mathcal{G})]$. This proves the statement of proposition 2.4 concerning characters of $\mathbb{T}$. The abelian variety associated to a $p$-new modular form $f$ is split multiplicative at $p$ if and only if $U_{p} f=f$. Moreover, the Atkin-Lehner involution at $p$ acts on a $p$-new modular form as $-U_{p}$, and acts on $\mathcal{M}$ as $\Gamma_{+} / \Gamma$. This concludes the proof of proposition 2.4 .

\section{Modular parametrizations, I}

We now make a specific choice of the operator $\eta_{f}$ (where $f$ is the newform of level $N$ attached to $E$ ) considered in the introduction, that will be used in formulating the results in the sequel of the paper.

As stated in lemma 2.3, $\bar{\Gamma}$ can be identified with the homology group $H_{1}(\mathcal{G}, \mathbb{Z}) \subset$ $\mathbb{Z}[\mathcal{E}(\mathcal{G})]$. Thus, when convenient, we will tacitly view elements of $\bar{\Gamma}$ as contained 
in $\mathbb{Z}[\mathcal{E}(\mathcal{G})]$. The restriction of the pairing on $\mathbb{Z}[\mathcal{E}(\mathcal{G})]$ defined above to $\bar{\Gamma}$ yields the monodromy pairing (denoted in the same way by an abuse of notation)

$$
\langle,\rangle: \bar{\Gamma} \times \bar{\Gamma} \rightarrow \mathbb{Z}
$$

Let $\mathbb{Z}[\mathcal{E}(\mathcal{G})]^{f}$, resp. $\bar{\Gamma}^{f}$ be the submodule of $\mathbb{Z}[\mathcal{E}(\mathcal{G})]$, resp. $\bar{\Gamma}$ on which $\tilde{\mathbb{T}}$, resp. $\mathbb{T}$ acts via the character associated with $f$. Note that the quotient of $\mathbb{Z}[\mathcal{E}(\mathcal{G})]$ by $\bar{\Gamma}$ is torsion-free, and thus there is a canonical identification $\mathbb{Z}[\mathcal{E}(\mathcal{G})]^{f}=\bar{\Gamma}^{f}$. Let $e^{f}$ be a generator of $\bar{\Gamma}^{f} \simeq \mathbb{Z}$.

Define the "modular parametrizations"

$$
\pi_{*}: \bar{\Gamma} \rightarrow \bar{\Gamma}^{f}, \quad \pi^{*}: \bar{\Gamma}^{f} \rightarrow \bar{\Gamma}
$$

by $\pi_{*}(e):=\left\langle e, e^{f}\right\rangle e^{f}$ and $\pi^{*}\left(e^{f}\right):=e^{f}$. Since

$$
\left(\pi^{*} \circ \pi_{*}\right)^{2}=\left\langle e^{f}, e^{f}\right\rangle\left(\pi^{*} \circ \pi_{*}\right)
$$

we obtain that $\pi^{*} \circ \pi_{*}$ is equal to $\left\langle e^{f}, e^{f}\right\rangle \pi_{f}$, where $\pi_{f}$ is the idempotent of $\mathbb{T} \otimes \mathbb{Q}$ associated with $f$. From now on, we will assume that the operator $\eta_{f}$ is defined by

$$
\eta_{f}:=\pi^{*} \circ \pi_{*}
$$

so that the integer $n_{f}$ is equal to $\left\langle e^{f}, e^{f}\right\rangle$.

As observed in the introduction, the operator $\eta_{f}$ induces a map $\mathcal{N} \rightarrow \mathbb{Z}$, which is well-defined up to sign. Since $f$ has split multiplicative reduction at $p$, this map factors through a map $\mathcal{N}_{\text {sp }} \rightarrow \mathbb{Z}$. By an abuse of notation, we will indicate by $\eta_{f}$ both the above maps.

\section{Remark 2.5}

The module $\bar{\Gamma}$ can be identified with the character group associated with the reduction modulo $p$ of $\operatorname{Pic}^{0}(X)$, where $X$ is the Shimura curve considered in the introduction. As will be explained in section 4, the map $\pi^{*} \circ \pi_{*}$ on $\bar{\Gamma}$ is induced by functoriality from a modular parametrization $\operatorname{Pic}^{0}(X) \rightarrow E$.

\section{The p-adic L-function}

Let $\mathcal{O}_{n}$ denote the order of $K$ of conductor $c p^{n}, n \geq 0$. (We will usually write $\mathcal{O}$ instead of $\left.\mathcal{O}_{0}.\right)$ Equip the orders $\mathcal{O}_{n}$ with compatible orientations, i.e., with compatible algebra homomorphisms

$$
\begin{array}{cc}
\mathfrak{d}_{\ell}^{+}: \mathcal{O}_{n} \rightarrow \mathbb{Z} / \ell^{m} \mathbb{Z}, & \ell^{m} \| N^{+} p, \\
\mathfrak{d}_{\ell}^{-}: \mathcal{O}_{n} \rightarrow \mathbb{F}_{\ell^{2}}, & \ell \mid N^{-} .
\end{array}
$$

An algebra homomorphism of $\mathcal{O}_{n}$ into an oriented Eichler order $S$ of level $N^{+} p$ is called an oriented optimal embedding if it respects the orientation on $\mathcal{O}_{n}$ and on $S$, and does not extend to an embedding of a larger order into $S$. Consider pairs $\left(R_{\xi}, \xi\right)$, where $R_{\xi}$ is an oriented Eichler order of level $N^{+} p$ and $\xi$ is an element of $\operatorname{Hom}(K, B)$ which restricts to an oriented optimal embedding of $\mathcal{O}_{n}$ into $R_{\xi}$. A 
Gross point of conductor $c p^{n}(n \geq 0)$ is a pair as above, taken modulo the action of $B^{\times}$.

By our previous remarks, a Gross point can be viewed naturally as an element of the double coset space

$$
W:=\left(\hat{R}^{\times} \backslash \hat{B}^{\times} \times \operatorname{Hom}(K, B)\right) / B^{\times} .
$$

(See $[\mathrm{Gr}]$, sec. 3 for more details.) Strong approximation gives the identification

$$
W=(\overrightarrow{\mathcal{E}}(\mathcal{T}) \times \operatorname{Hom}(K, B)) / \Gamma_{+}
$$

By lemma 2.2 , there is a natural map of $\mathbb{Z}$-modules $\mathbb{Z}[W] \rightarrow \mathbb{Z}[\mathcal{E}(\mathcal{G})]$, where $\mathbb{Z}[W]$ is the module of finite formal $\mathbb{Z}$-linear combinations of elements of $W$. The Hecke algebra $\tilde{\mathbb{T}}$ of $\mathbb{Z}[\mathcal{E}(\mathcal{G})]$ acts naturally also on $\mathbb{Z}[W]$ (see [BD1], sec. 1.5), in such a way that the above map is $\tilde{\mathbb{T}}$-equivariant.

The group $\mathbf{G}_{n}=\operatorname{Pic}\left(\mathcal{O}_{n}\right)=\hat{\mathcal{O}}_{n}^{\times} \backslash \hat{K}^{\times} / K^{\times}$acts simply transitively on the Gross points of conductor $c p^{n}$ by the rule

$$
\sigma\left(R_{\xi}, \xi\right):=\left(R_{\xi} * \hat{\xi}(\sigma)^{-1}, \xi\right)
$$

where $\hat{\xi}$ denotes the extension of $\xi$ to a map from $\hat{K}$ to $\hat{B}$.

Now, fix a Gross point $P_{0}=\left(R_{0}, \xi_{0}\right)\left(\bmod B^{\times}\right)$of conductor $c$. By the above identification, $P_{0}$ corresponds to a pair $\left(\vec{e}_{0}, \xi_{0}\right) \in \overrightarrow{\mathcal{E}}(\mathcal{T}) \times \operatorname{Hom}(K, B)$, modulo the action of $\Gamma_{+}$. As above, the origin $v_{0}$ of $\vec{e}_{0}$ determines an orientation of $\mathcal{T}$. Let $\vec{e}$ be one of the $p$ oriented edges of $\mathcal{T}$ originating from $\vec{e}_{0}$. All the Gross points corresponding to pairs $\left(\vec{e}, \xi_{0}\right)$ as above have conductor $c p$, except for one, which has conductor $c$. Fix an end

$$
\left(\vec{e}_{0}, \vec{e}_{1}, \ldots, \vec{e}_{n}, \ldots\right)
$$

such that $\left(\vec{e}_{1}, \xi_{0}\right)$ defines a Gross point of conductor $c p$. Then, $\left(\vec{e}_{n}, \xi_{0}\right)$ defines a Gross point $P_{n}$ of conductor $c p^{n}$, for all $n \geq 0$.

Denote by $\operatorname{Norm}_{H_{n+1} / H_{n}}$ the norm operator $\sum_{g \in \operatorname{Gal}\left(H_{n+1} / H_{n}\right)} g$.

\section{Lemma 3.1}

1) Let $u=\frac{1}{2} \# \mathcal{O}^{\times}$. The equality

$$
U_{p} P_{0}=u \operatorname{Norm}_{H_{1} / H_{0}} P_{1}+\sigma_{\mathfrak{p}} P_{0}
$$

holds in $\mathbb{Z}[W]$ for a prime $\mathfrak{p}$ above $p$, where $\sigma_{\mathfrak{p}} \in \operatorname{Gal}\left(H_{0} / K\right)$ denotes the image of $\mathfrak{p}$ by the Artin map.

2) For $n \geq 1$,

$$
U_{p} P_{n}=\operatorname{Norm}_{H_{n+1} / H_{n}} P_{n+1}
$$

Proof. It follows from the definition of the operator $U_{p}$ (see [BD1], sec. 1.5) and the action of $\operatorname{Pic}\left(\mathcal{O}_{n}\right)$ on the Gross points.

The picture below, drawn in the case $p=2$, illustrates geometrically the relation between the Galois action and the action of the Hecke correspondence $U_{p}$. 
By lemma 2.3 , the natural map from $\mathbb{Z}[W]$ to $\mathbb{Z}[\mathcal{E}(\mathcal{G})]$ induces maps from $\mathbb{Z}[W]$ to the modules $\mathcal{N}$ and $\mathcal{N}_{\mathrm{sp}}$. These maps are Hecke-equivariant.

The Gross points $P_{n}$ give rise to a $p$-adic distribution on $\mathbf{G}_{\infty}$ with values in the module $\mathcal{N}_{\mathrm{sp}}$ as follows. Given $g \in \mathbf{G}_{n}$, denote by $e_{n}^{g}$ the natural image of $P_{n}^{g}$ in $\mathcal{N}_{\mathrm{sp}}$. For $n \geq 0$, define the truncated $p$-adic $L$-function

$$
\mathcal{L}_{p, n}\left(\mathcal{N}_{\mathrm{sp}} / K\right):=\sum_{g \in \mathbf{G}_{n}} e_{n}^{g} \cdot g^{-1} \in \mathcal{N}_{\mathrm{sp}} \otimes \mathbb{Z}\left[\mathbf{G}_{n}\right] .
$$

Note that $\mathcal{L}_{p, n}\left(\mathcal{N}_{\mathrm{sp}} / K\right)$ is well-defined up to multiplication by elements of $\mathbf{G}_{n}$.

For $n \geq 1$, let $\nu_{n}: \mathbb{Z}\left[\mathbf{G}_{n}\right] \rightarrow \mathbb{Z}\left[\mathbf{G}_{n-1}\right]$ be the natural projection of groups rings.

\section{Lemma 3.2}

1) The equality

$$
\nu_{1}\left(\mathcal{L}_{p, 1}\left(\mathcal{N}_{\mathrm{sp}} / K\right)\right)=u^{-1}\left(1-\sigma_{\mathfrak{p}}\right) \mathcal{L}_{p, 0}\left(\mathcal{N}_{\mathrm{sp}} / K\right)
$$

holds in $\mathcal{N}_{\mathrm{sp}} \otimes \mathbb{Z}\left[\mathbf{G}_{0}\right]$.

2) For $n \geq 2$, the equality

$$
\nu_{n}\left(\mathcal{L}_{p, n}\left(\mathcal{N}_{\mathrm{sp}} / K\right)\right)=\mathcal{L}_{p, n-1}\left(\mathcal{N}_{\mathrm{sp}} / K\right)
$$

holds in $\mathcal{N}_{\mathrm{sp}} \otimes \mathbb{Z}\left[\mathbf{G}_{n-1}\right]$.

Proof. By proposition 2.4, the operator $U_{p}$ acts as +1 on $\mathcal{N}_{\mathrm{sp}}$. The claim follows from lemma 3.1 and the fact that $\mathcal{N}_{\mathrm{sp}}$ is torsion-free.

Define the $p$-adic $L$-function attached to $\mathcal{N}_{\text {sp }}$ to be

$$
\mathcal{L}_{p}\left(\mathcal{N}_{\mathrm{sp}} / K\right):=\lim _{n} \mathcal{L}_{p, n}\left(\mathcal{N}_{\mathrm{sp}} / K\right) \in \mathcal{N}_{\mathrm{sp}} \otimes \mathbb{Z} \llbracket \mathbf{G}_{\infty} \rrbracket
$$

We now define the $p$-adic $L$-function attached to $E$. Observe that the maximal quotient $\bar{\Gamma}_{f}$ of $\bar{\Gamma}$ on which $\mathbb{T}$ acts via the character associated with $f$ is isomorphic to $\mathbb{Z}$. Let $e_{f}$ be a generator of $\bar{\Gamma}_{f}$. The monodromy pairing on $\bar{\Gamma}$ induces a $\mathbb{Z}$-valued pairing on $\bar{\Gamma}^{f} \times \bar{\Gamma}_{f}$. Write $\hat{c}_{p}$ for the positive integer $\left|\left\langle e^{f}, e_{f}\right\rangle\right|$.

\section{Lemma 3.3}

The element $\left(\eta_{f} \otimes \mathrm{id}\right)\left(\mathcal{L}_{p}\left(\mathcal{N}_{\mathrm{sp}} / K\right)\right) \in \mathbb{Z} \llbracket \mathbf{G}_{\infty} \rrbracket$ is divisible by $\hat{c}_{p}$.

Proof. Consider the maps

$$
\tilde{\pi}_{*}: \mathbb{Z}[\mathcal{E}(\mathcal{G})] \rightarrow \mathbb{Z}[\mathcal{E}(\mathcal{G})]^{f}, \quad \tilde{\pi}^{*}: \mathbb{Z}[\mathcal{E}(\mathcal{G})]^{f} \rightarrow \mathbb{Z}[\mathcal{E}(\mathcal{G})]
$$

defined by $\tilde{\pi}_{*}(e):=\left\langle e, e^{f}\right\rangle e^{f}$ and $\tilde{\pi}^{*}\left(e^{f}\right):=e^{f}$. (The modular parametrizations $\pi_{*}$ and $\pi^{*}$ introduced in section 2 are obtained from these maps by restriction.) Hence, $\tilde{\eta}_{f}:=\tilde{\pi}^{*} \circ \tilde{\pi}_{*}$ is an element of $\tilde{\mathbb{T}}$, equal to $\left\langle e^{f}, e^{f}\right\rangle \tilde{\pi}_{f}$, where $\tilde{\pi}_{f}$ is the idempotent in $\tilde{\mathbb{T}} \otimes \mathbb{Q}$ associated with $f$. We have a commutative diagram

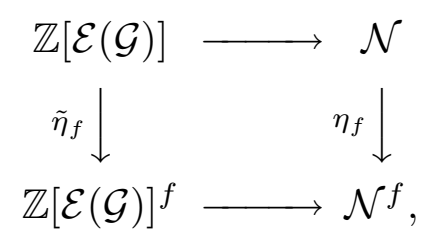


where the upper horizontal map is defined in lemma 2.3, and the lower horizontal map is the restriction of the upper one. Note that $\mathcal{N}^{f}$ is equal to $\operatorname{Hom}\left(\bar{\Gamma}_{f}, \mathbb{Z}\right)$, and therefore is generated by the homomorphism $e_{f} \mapsto 1$. With our choices of generators for $\mathbb{Z}[\mathcal{E}(\mathcal{G})]^{f}$ and $\mathcal{N}^{f}$, the lower map of the above diagram is described as multiplication by the integer $\left\langle e^{f}, e_{f}\right\rangle$. The proof of lemma 3.2 also shows that mapping the Gross points of conductor $c p^{n}$ to $\mathbb{Z}[\mathcal{E}(\mathcal{G})]^{f}$ by the map $\tilde{\eta}_{f}$ yields a $p$-adic distribution in $\mathbb{Z}[\mathcal{E}(\mathcal{G})]^{f} \otimes \mathbb{Z} \llbracket \mathbf{G}_{\infty} \rrbracket$. By the above diagram, the image of this distribution in $\mathcal{N}^{f} \otimes \mathbb{Z} \llbracket \mathbf{G}_{\infty} \rrbracket$ is equal to $\left(\eta_{f} \otimes \mathrm{id}\right)\left(\mathcal{L}_{p}\left(\mathcal{N}_{\mathrm{sp}} / K\right)\right)$. This proves the lemma.

Remark 3.4 In section 4, we will show that the integers $\hat{c}_{p}$ and $c_{p}$ are equal.

Define the $p$-adic $L$-function attached to $E$ to be

$$
\mathcal{L}_{p}(E / K)=\hat{c}_{p}^{-1}\left(\eta_{f} \otimes \mathrm{id}\right)\left(\mathcal{L}_{p}\left(\mathcal{N}_{\mathrm{sp}} / K\right)\right) \in \mathbb{Z} \llbracket \mathbf{G}_{\infty} \rrbracket
$$

Observe that $\mathcal{L}_{p}\left(\mathcal{N}_{\mathrm{sp}} / K\right)$ and $\mathcal{L}_{p}(E / K)$ are well-defined up to multiplication by elements of $\mathbf{G}_{\infty}$.

Recall the quantities $\Omega_{f}$ and $d$ defined in the introduction.

\section{Theorem 3.5}

Let $\chi: \mathbf{G}_{\infty} \rightarrow \mathbb{C}^{\times}$be a finite order character of conductor $c p^{n}$, with $n \geq 1$. Then the equality

$$
\left|\chi\left(\mathcal{L}_{p}(E / K)\right)\right|^{2}=\frac{L(E / K, \chi, 1)}{\Omega_{f}} \sqrt{d} \cdot\left(n_{f} u\right)^{2}
$$

holds.

Proof. See [Gr], [Dag], and [BD1], section 2.10.

\section{Remark 3.6}

1) Theorem 3.5 suggests that $\mathcal{L}_{p}(E / K)$ should really be viewed as the square root of a $p$-adic $L$-function, and hence we should define the anticyclotomic $p$-adic $L$-function of $E$ to be $\mathcal{L}_{p}(E / K) \otimes \mathcal{L}_{p}(E / K)^{*}$, where $*$ denotes the involution of $\mathbb{Z} \llbracket \mathbf{G}_{\infty} \rrbracket$ given on group-like elements by $g \mapsto g^{-1}$. See section 2.7 of [BD1] for more details.

2) More generally, the $p$-adic $L$-function $\mathcal{L}_{p}\left(\mathcal{N}_{\mathrm{sp}} / K\right)$ interpolates special values of the complex $L$-series attached to the modular forms on $\mathbb{T}_{\mathrm{sp}}$ (described in proposition $2.4)$.

Let $\sigma_{\mathfrak{p}}$ be as in lemma 3.1. Denote by $H$ the subextension of $H_{0}$ which is fixed by $\sigma_{\mathfrak{p}}$, and set

$$
\begin{aligned}
& G_{n}:=\operatorname{Gal}\left(H_{n} / H\right), \quad G_{\infty}:=\operatorname{Gal}\left(H_{\infty} / H\right), \\
& \Sigma:=\operatorname{Gal}\left(H_{0} / H\right)=G_{0}, \quad \Delta:=\operatorname{Gal}(H / K) .
\end{aligned}
$$

Note the exact sequences of Galois groups

$$
\begin{gathered}
0 \rightarrow G_{n} \rightarrow \mathbf{G}_{n} \rightarrow \Delta \rightarrow 0 \\
0 \rightarrow G_{\infty} \rightarrow \mathbf{G}_{\infty} \rightarrow \Delta \rightarrow 0
\end{gathered}
$$


The group $\Delta$ is naturally identified with the Picard group $\operatorname{Pic}\left(\mathcal{O}\left[\frac{1}{p}\right]\right)$, and $G_{\infty}$ is

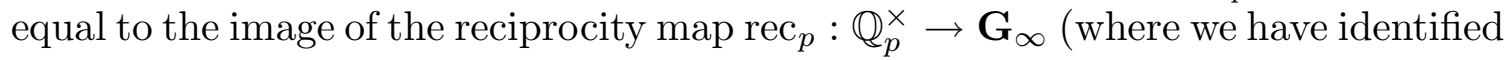
$\mathbb{Q}_{p}^{\times}$with $\left.K_{\mathfrak{p}}^{\times}\right)$. Let $I$ be the kernel of the augmentation map $\mathbb{Z} \llbracket \mathbf{G}_{\infty} \rrbracket \rightarrow \mathbb{Z}$, and let $I_{\Delta}$ be the kernel of the augmentation map $\mathbb{Z} \llbracket \mathbf{G}_{\infty} \rrbracket \rightarrow \mathbb{Z}[\Delta]$.

\section{Lemma 3.7}

i) $\mathcal{L}_{p}\left(\mathcal{N}_{\mathrm{sp}} / K\right)$ belongs to $\mathcal{N}_{\mathrm{sp}} \otimes I_{\Delta}$.

ii) $\mathcal{L}_{p}(E / K)$ belongs to $I_{\Delta}$.

Proof. There are canonical isomorphisms

$$
\mathbb{Z} \llbracket \mathbf{G}_{\infty} \rrbracket / I_{\Delta}=\mathbb{Z}\left[\mathbf{G}_{n}\right] / I_{\Delta, n}=\mathbb{Z}[\Delta],
$$

where $I_{\Delta, n}$ is the natural image of $I_{\Delta}$ in $\mathbb{Z}\left[\mathbf{G}_{n}\right]$. By lemma 3.2, the image of $\mathcal{L}_{p}\left(\mathcal{N}_{\mathrm{sp}} / K\right)$ in $\mathcal{N}_{\mathrm{sp}} \otimes\left(\mathbb{Z} \llbracket \mathbf{G}_{\infty} \rrbracket / I_{\Delta}\right)$ is equal to the image of $\mathcal{L}_{p, 1}\left(\mathcal{N}_{\mathrm{sp}} / K\right)$ in $\mathcal{N}_{\mathrm{sp}} \otimes$ $\left(\mathbb{Z}\left[\mathbf{G}_{1}\right] / I_{\Delta, 1}\right)=\mathcal{N}_{\mathrm{sp}} \otimes \mathbb{Z}[\Delta]$. The first part of the lemma now follows from lemma $3.2,1)$. The second part follows directly from the first.

Since $I_{\Delta}$ is contained in $I$, the element $\mathcal{L}_{p}\left(\mathcal{N}_{\mathrm{sp}} / K\right)$ belongs to $\mathcal{N}_{\mathrm{sp}} \otimes I$ and $\mathcal{L}_{p}(E / K)$ belongs to $I$. Denote by

$$
\mathcal{L}_{p}^{\prime}\left(\mathcal{N}_{\mathrm{sp}} / K\right), \quad \mathcal{L}_{p}^{\prime}\left(\mathcal{N}_{\mathrm{sp}} / H\right)
$$

the natural image of $\mathcal{L}_{p}\left(\mathcal{N}_{\mathrm{sp}} / K\right)$ in $\mathcal{N}_{\mathrm{sp}} \otimes I / I^{2}=\mathcal{N}_{\mathrm{sp}} \otimes \mathbf{G}_{\infty}$ and $\mathcal{N}_{\mathrm{sp}} \otimes I_{\Delta} / I_{\Delta}^{2}=$ $\mathcal{N}_{\mathrm{sp}} \otimes \mathbb{Z}[\Delta] \otimes G_{\infty}$, respectively. Likewise, let

$$
\mathcal{L}_{p}^{\prime}(E / K), \quad \mathcal{L}_{p}^{\prime}(E / H)
$$

be the natural image of $\mathcal{L}_{p}(E / K)$ in $I / I^{2}=\mathbf{G}_{\infty}$ and $I_{\Delta} / I_{\Delta}^{2}=\mathbb{Z}[\Delta] \otimes G_{\infty}$, respectively. The above elements should be viewed as derivatives of $p$-adic $L$-functions at the central point.

In order to carry out the calculations of the next sections, it is useful to observe that the derivatives $\mathcal{L}_{p}^{\prime}\left(\mathcal{N}_{\mathrm{sp}} / K\right)$ and $\mathcal{L}_{p}^{\prime}\left(\mathcal{N}_{\mathrm{sp}} / H\right)$ can be expressed in terms of the derivatives of certain partial $p$-adic $L$-functions. Set $h:=\#(\Delta)$. Fix Gross points of conductor $c$

$$
P_{0}=P_{0}^{1}, \ldots, P_{0}^{h},
$$

corresponding to pairs $\left(R_{0}^{i}, \xi_{0}^{i}\right), i=1, \ldots, h$, which are representatives for the $\Sigma$ orbits of the Gross points of conductor $c$. Writing $\left[P_{0}^{i}\right]$ for the $\Sigma$-orbit of $P_{0}^{i}$, let $\delta_{i}$ be the element of $\Delta$ such that

$$
\left[\delta_{i} P_{0}^{1}\right]=\left[P_{0}^{i}\right]
$$

Suppose that $P_{0}^{i}$ corresponds to a pair $\left(\vec{e}_{0}(i), \xi_{0}^{i}\right) \in \overrightarrow{\mathcal{E}}(\mathcal{T}) \times \operatorname{Hom}(K, B)$, modulo the action of $\Gamma_{+}$. Fix ends

$$
\left(\vec{e}_{0}(i), \vec{e}_{1}(i), \ldots, \vec{e}_{n}(i), \ldots\right)
$$

such that $\left(\vec{e}_{1}(i), \xi_{0}^{i}\right)$ defines a Gross point of conductor $c p$. Thus, $\left(\vec{e}_{n}(i), \xi_{0}^{i}\right)$ defines a Gross point $P_{n}^{i}$ of conductor $c p^{n}$, for all $n \geq 0$. For $g \in G_{n}$, let $e_{n}(i)^{g}$ denote the natural image of $\left(P_{n}^{i}\right)^{g}$ in $\mathcal{N}_{\mathrm{sp}}$. Let

$$
\mathcal{L}_{p, n}\left(\mathcal{N}_{\mathrm{sp}} / H, P_{0}^{i}\right):=\sum_{g \in G_{n}} e_{n}(i)^{g} \cdot g^{-1} \in \mathcal{N}_{\mathrm{sp}} \otimes \mathbb{Z}\left[G_{n}\right] .
$$


The proof of lemma 3.2 also shows that the elements $\mathcal{L}_{p, n}\left(\mathcal{N}_{\mathrm{sp}} / H, P_{0}^{i}\right)$ are compatible under the maps induced by the natural projections of group rings. Thus, we may define the partial $p$-adic $L$-function attached to $\mathcal{N}_{\mathrm{sp}}$ and $P_{0}^{i}$ to be

$$
\mathcal{L}_{p}\left(\mathcal{N}_{\mathrm{sp}} / H, P_{0}^{i}\right):=\lim _{\overleftarrow{n}} \mathcal{L}_{p, n}\left(\mathcal{N}_{\mathrm{sp}} / H, P_{0}^{i}\right) \in \mathcal{N}_{\mathrm{sp}} \otimes \mathbb{Z} \llbracket G_{\infty} \rrbracket
$$

Observe that $\mathcal{L}_{p}\left(\mathcal{N}_{\mathrm{sp}} / H, P_{0}^{i}\right)$ depends only on the $\Sigma$-orbit of $P_{0}^{i}$, up to multiplication by elements of $G_{\infty}$.

Let $I_{H}$ be the kernel of the augmentation map $\mathbb{Z} \llbracket G_{\infty} \rrbracket \rightarrow \mathbb{Z}$. Like in the proof of lemma 3.7, one checks that $\mathcal{L}_{p}\left(\mathcal{N}_{\mathrm{sp}} / H, P_{0}^{i}\right)$ belongs to $I_{H}$. Write $\mathcal{L}_{p}^{\prime}\left(\mathcal{N}_{\mathrm{sp}} / H, P_{0}^{i}\right)$ for the natural image of $\mathcal{L}_{p}\left(\mathcal{N}_{\mathrm{sp}} / H, P_{0}^{i}\right)$ in $\mathcal{N}_{\mathrm{sp}} \otimes I_{H} / I_{H}^{2}=\mathcal{N}_{\mathrm{sp}} \otimes G_{\infty}$. Thus,

$$
\mathcal{L}_{p}^{\prime}\left(\mathcal{N}_{\mathrm{sp}} / H, P_{0}^{i}\right)=\lim _{\overleftarrow{n}} \mathcal{L}_{p, n}^{\prime}\left(\mathcal{N}_{\mathrm{sp}} / H, P_{0}^{i}\right)
$$

where

$$
\mathcal{L}_{p, n}^{\prime}\left(\mathcal{N}_{\mathrm{sp}} / H, P_{0}^{i}\right)=\sum_{g \in G_{n}} e_{n}(i)^{g} \otimes g^{-1}
$$

We obtain directly:

\section{Lemma 3.8}

i)

$$
\mathcal{L}_{p}^{\prime}\left(\mathcal{N}_{\mathrm{sp}} / K\right)=\sum_{i=1}^{h} \mathcal{L}_{p}^{\prime}\left(\mathcal{N}_{\mathrm{sp}} / H, P_{0}^{i}\right)
$$

ii)

$$
\mathcal{L}_{p}^{\prime}\left(\mathcal{N}_{\mathrm{sp}} / H\right)=\sum_{i=1}^{h} \mathcal{L}_{p}^{\prime}\left(\mathcal{N}_{\mathrm{sp}} / H, P_{0}^{i}\right) \cdot \delta_{i}^{-1}
$$

\section{The theory of p-adic uniformization of Shimura curves}

For more details on the results stated in this section, the reader is referred to $[\mathrm{BC}],[\mathrm{Cer}],[\mathrm{Dr}],[\mathrm{GVdP}]$ and [BD2].

Let $\mathcal{B}$ be the indefinite quaternion algebra over $\mathbb{Q}$ of discriminant $N^{-} p$, and let $\mathcal{R}$ be an Eichler order of $\mathcal{B}$ of level $N^{+}$. Denote by $X$ the Shimura curve over $\mathbb{Q}$ associated with the order $\mathcal{R}$. We refer the reader to [BC] and [BD2], section 4 for the definition of $X$ via moduli. Here we content ourselves with recalling Cerednik's theorem, which describes a rigid-analytic uniformization of $X$. Write

$$
\mathcal{H}_{p}:=\mathbb{C}_{p}-\mathbb{Q}_{p}
$$

for the $p$-adic upper half plane. The group $\mathrm{GL}_{2}\left(\mathbb{Q}_{p}\right)$ acts (on the left) on $\mathcal{H}_{p}$ by linear fractional transformations. Thus, fixing an isomorphism

$$
\psi: B_{p} \rightarrow M_{2}\left(\mathbb{Q}_{p}\right)
$$


induces an action of $\Gamma$ on $\mathcal{H}_{p}$. This action is discontinuous, and the rigid-analytic quotient $\Gamma \backslash \mathcal{H}_{p}$ defines the $\mathbb{C}_{p}$-points of a non-singular curve $\mathcal{X}$ over $\mathbb{Q}_{p}$. The curves $X$ and $\mathcal{X}$ are equipped with the action of Hecke algebras $\mathbb{T}_{X}$ and $\mathbb{T}_{\mathcal{X}}$, respectively $([\mathrm{BC}],[\mathrm{BD} 1])$.

By lemma 2.1, the action of $\Gamma_{+} / \Gamma$ induces an involution $W$ of $\mathcal{X}$. Let $\mathbb{Q}_{p^{2}}$ be the unique unramified quadratic extension of $\mathbb{Q}_{p}$ contained in $\mathbb{C}_{p}$, and let $\tau$ be the generator of $\operatorname{Gal}\left(\mathbb{Q}_{p^{2}} / \mathbb{Q}_{p}\right)$. Denote by $\downarrow \in H^{1}(\langle\tau\rangle, \operatorname{Aut}(\mathcal{X}))$ the class of the cocycle mapping $\tau$ to $W$, and write $\mathcal{X}^{\natural}$ for the curve over $\mathbb{Q}_{p}$ obtained by twisting $\mathcal{X}$ by $\downarrow$.

\section{Theorem 4.1 (Cerednik)}

There is a Hecke-equivariant isomorphism $X \simeq \mathcal{X}^{\natural}$ of curves over $\mathbb{Q}_{p}$. In particular, $X$ and $\mathcal{X}$ are isomorphic over $\mathbb{Q}_{p^{2}}$.

Proof. See [Cer], [Dr], [BC].

Building on theorem 4.1, the results in $[\mathrm{GVdP}]$ yield a rigid-analytic description of the jacobian of $X$. If $D=P_{1}+\cdots+P_{r}-Q_{1}-\cdots-Q_{r} \in \operatorname{Div}^{0}\left(\mathcal{H}_{p}\right)$ is a divisor of degree zero on $\mathcal{H}_{p}$, define the theta function

$$
\vartheta(z ; D)=\prod_{\epsilon \in \Gamma} \frac{\left(z-\epsilon P_{1}\right) \cdots\left(z-\epsilon P_{r}\right)}{\left(z-\epsilon Q_{1}\right) \cdots\left(z-\epsilon Q_{r}\right)}
$$

Write $\bar{\delta}$ for the natural image in $\bar{\Gamma}$ of an element $\delta$ of $\Gamma$. For all $\delta$ in $\Gamma$, the above theta function satisfies the functional equation

$$
\vartheta(\delta z ; D)=\phi_{D}(\bar{\delta}) \vartheta(z ; D)
$$

where $\phi_{D}$ is an element of $\operatorname{Hom}\left(\bar{\Gamma}, \mathbb{C}_{p}^{\times}\right)=\mathcal{N} \otimes \mathbb{C}_{p}^{\times}$which does not depend on $z$. For $\gamma \in \Gamma$, the number $\phi_{(\gamma z)-(z)}(\bar{\delta})$ does not depend on the choice of $z \in \mathcal{H}_{p}$, and depends only on the image of $\gamma$ in $\bar{\Gamma}$. This gives rise to a pairing

$$
[,]: \bar{\Gamma} \times \bar{\Gamma} \rightarrow \mathbb{Q}_{p}^{\times}
$$

The pairing [, ] is bilinear and symmetric. The next proposition explains the relation between $[$,$] and the monodromy pairing \langle\rangle:, \bar{\Gamma} \times \bar{\Gamma} \rightarrow \mathbb{Z}$ defined in section 2 .

\section{Proposition 4.2}

The pairings $\langle$,$\rangle and \operatorname{ord}_{p} \circ[$,$] are equal.$

Proof. See $[\mathrm{M}]$, th. 7.6 .

It follows that $\operatorname{ord}_{p} \circ[$,$] is positive definite, so that themap$

$$
j: \bar{\Gamma} \rightarrow \mathcal{N} \otimes \mathbb{Q}_{p}^{\times}
$$

induced by [, ] is injective and has discrete image. Set $\Lambda:=j(\bar{\Gamma})$. Given a divisor $D$ of degree zero on $\mathcal{X}\left(\mathbb{C}_{p}\right)=\Gamma \backslash \mathcal{H}_{p}$, let $\tilde{D}$ denote an arbitrary lift to a degree zero divisor on $\mathcal{H}_{p}$. The automorphy factor $\phi_{\tilde{D}}$ depends on the choice of the lift $\tilde{D}$, but 
its image in $\left(\mathcal{N} \otimes \mathbb{C}_{p}^{\times}\right) / \Lambda$ depends only on $D$. Thus, the assignment $D \mapsto \phi_{\tilde{D}}$ gives a well-defined map from $\operatorname{Div}^{0}\left(\mathcal{X}\left(\mathbb{C}_{p}\right)\right)$ to $\left(\mathcal{N} \otimes \mathbb{C}_{p}^{\times}\right) / \Lambda$.

\section{Proposition 4.3}

The map $\operatorname{Div}^{0}\left(\mathcal{X}\left(\mathbb{C}_{p}\right)\right) \rightarrow\left(\mathcal{N} \otimes \mathbb{C}_{p}^{\times}\right) / \Lambda$ defined above is trivial on the group of principal divisors, and induces a Hecke-equivariant isomorphism from the $\mathbb{C}_{p}$-points of the jacobian $\mathcal{J}$ of $\mathcal{X}$ to $\left(\mathcal{N} \otimes \mathbb{C}_{p}^{\times}\right) / \Lambda$.

Proof. See [GVdP], VI.2 and VIII.4, and also [BC], ch. III.

Let

$$
\Phi: \mathcal{N} \otimes \mathbb{C}_{p}^{\times} \rightarrow \mathcal{J}\left(\mathbb{C}_{p}\right)
$$

stand for the map induced by (the inverse of) the isomorphism defined in proposition 4.3.

\section{Modular parametrizations, II}

The map $\eta_{f}: \mathcal{N} \rightarrow \mathbb{Z}$ defined in section 2 induces a map

$$
\eta_{f} \otimes \mathrm{id}: \mathcal{N} \otimes \mathbb{C}_{p}^{\times} \rightarrow \mathbb{C}_{p}^{\times}
$$

The Jacquet-Langlands correspondence [JL] implies that the quotient abelian variety $\eta_{f} J$ is an elliptic curve $\mathbb{Q}$-isogenous to $E$. From now on, we assume that $E=\eta_{f} J$ is the strong Weil curve for the parametrization by the Shimura curve $X$. By an abuse of notation, we denote by $\eta_{f}$ also the surjective map

$$
J\left(\mathbb{C}_{p}\right) \rightarrow E\left(\mathbb{C}_{p}\right)
$$

induced by $\eta_{f}$.

Let $\Lambda^{f}$ be the submodule of $\Lambda$ on which $\mathbb{T}$ acts via the character $\phi_{f}$.

\section{Proposition 4.4}

The kernel $q^{\mathbb{Z}}$ of $\Phi_{\text {Tate }}$ is canonically equal to the module $\Lambda^{f}$, and the following diagram

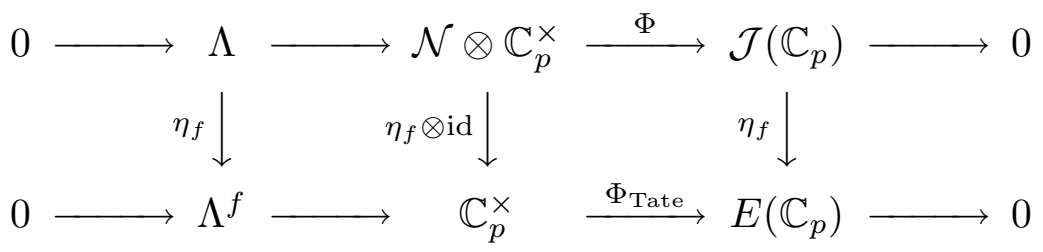

is Hecke-equivariant and commutes up to sign.

Proof. The right-most square in the above diagram is a consequence of proposition 4.3, combined with theorem 4.1 and the fact that $f$ is split-multiplicative at $p$. In order to obtain the left-most square, it is enough to prove that the kernel of $\Phi_{\text {Tate }}$ is equal to $\Lambda^{f}$. Note that the target $\mathbb{C}_{p}^{\times}=\mathcal{N}^{f} \otimes \mathbb{C}_{p}^{\times}$of the map $\eta_{f} \otimes \mathrm{id}$ is naturally a submodule of $\mathcal{N} \otimes \mathbb{C}_{p}^{\times}$, since the quotient of $\mathcal{N}$ by $\mathcal{N}^{f}$ is torsion-free. By definition, $E\left(\mathbb{C}_{p}\right)$ may similarly be viewed as an abelian subvariety of $\mathcal{J}\left(\mathbb{C}_{p}\right)$. It follows that $\Phi_{\text {Tate }}$ can be described as the restriction of $\Phi$ to $\mathbb{C}_{p}^{\times}$. In particular, $\operatorname{ker}\left(\Phi_{\text {Tate }}\right)$ is equal to $\Lambda \cap \mathbb{C}_{p}^{\times}$. In turn, this last module is equal to $\Lambda^{f}$. 


\section{Corollary 4.5}

The integer $\hat{c}_{p}=\left|\left\langle e^{f}, e_{f}\right\rangle\right|$ (introduced in lemma 3.3) is equal to $c_{p}$.

Proof. Working through the definition of the maps in the diagram of proposition 4.4 shows that $\left[e^{f}, e_{f}\right]$ is equal to $q^{ \pm 1}$. The claim follows from proposition 4.2 .

\section{5 p-adic Shintani cycles and special values of complex L- functions}

Let $P_{0}=\left(R_{0}, \xi_{0}\right)\left(\bmod B^{\times}\right)$be a Gross point of conductor $c$. The point $P_{0}$ determines a $p$-adic cycle $\mathfrak{c}\left(P_{0}\right) \in \bar{\Gamma}$ in the following way. By strong approximation, we may assume that the representative $\left(R_{0}, \xi_{0}\right)$ for $P_{0}$ is such that the oriented orders $R_{0}\left[\frac{1}{p}\right]$ and $R\left[\frac{1}{p}\right]$ are equal. Thus, $\xi_{0}$ induces an embedding of $\mathcal{O}\left[\frac{1}{p}\right]$ into $R\left[\frac{1}{p}\right]$, which we still denote by $\xi_{0}$. The image by $\xi_{0}$ of a fundamental $p$-unit in $\mathcal{O}\left[\frac{1}{p}\right]$ having norm of even $p$-adic valuation determines an element $\gamma=\gamma\left(P_{0}\right)$ of $\Gamma$. This element is well-defined up to conjugation and up to inversion, and up to multiplication by the image of torsion elements of $\mathcal{O}^{\times}$.

More explicitly, write $k$ for the order of $\sigma_{\mathfrak{p}}$ in $\operatorname{Pic}(\mathcal{O})$ (where $\sigma_{\mathfrak{p}}$ is as in lemma $3.1)$, and set $\mathfrak{p}^{k}=(v)$ with $v \in \mathcal{O}$. Let $\iota$ be 1 , resp. 2 if $k$ is even, resp. odd. Then $\gamma$ is the image of $\xi_{0}(v)^{\iota}$ in $\Gamma$.

Definition. The $p$-adic Shintani cycle $\mathfrak{c}=\mathfrak{c}\left(P_{0}\right)$ attached to $P_{0}$ is the natural image of $\gamma$ in $\bar{\Gamma}$.

This terminology is justified in the remark 5.4 below. Observe that $\mathfrak{c}$ is well-defined up to sign.

Denote by $\mathbb{Z}[\mathcal{E}(\mathcal{G})]_{\text {sp }}$ the maximal torsion-free quotient of $\mathbb{Z}[\mathcal{E}(\mathcal{G})] /(w+1) \mathbb{Z}[\mathcal{E}(\mathcal{G})]$, where $w$ is any element of $\Gamma_{+}-\Gamma$. Recall the element $\tilde{\eta}_{f} \in \tilde{\mathbb{T}}$ defined in the proof of lemma 3.3, mapping to $\eta_{f}$ by the natural projection $\tilde{\mathbb{T}} \rightarrow \mathbb{T}$. The next lemma relates the $p$-adic cycle $\mathfrak{c}$ to the image in $\mathcal{N}_{\mathrm{sp}}$ of the Gross point $P_{0}$.

\section{Lemma 5.1}

The natural images in $\mathbb{Z}[\mathcal{E}(\mathcal{G})]_{\mathrm{sp}}$ of $\mathfrak{c}$ and $\sum_{\sigma \in \Sigma} \iota P_{0}^{\sigma}$ are equal. In particular, $\eta_{f} \mathfrak{c}$ is equal to the image of $\sum_{\sigma \in \Sigma} \iota\left(\tilde{\eta}_{f} P_{0}^{\sigma}\right)$ in $\mathbb{Z}[\mathcal{E}(\mathcal{G})]$.

Proof. (In order to visualize the geometric content of this proof, the reader may find it helpful to refer to the picture in section 3.) Set $P_{i}:=\sigma_{\mathfrak{p}}^{i} P_{0}$, for $i=0, \ldots, k-1$. By part 1 of lemma 3.1 and the definition of the action of $U_{p}$ on the Bruhat-Tits tree, we can fix representatives $\left(\vec{e}_{i}, \xi_{0}\right)$ for the Gross points $P_{i}$ so that the $\vec{e}_{i}$ are consecutive oriented edges of $\mathcal{T}$. With notations as at the beginning of this section, let $\gamma_{+} \in \Gamma_{+}$be the image of $\xi_{0}(v)$. Thus, $\gamma=\gamma_{+}^{\iota}$. Call $v_{0}$ the origin of $\vec{e}_{0}$. If $\iota=1$, 
the even vertex of the edge $\vec{e}_{k-1}$ is equal to $\gamma v_{0}$. If $\iota=2$, i.e., $\gamma_{+}$belongs to $\Gamma_{+}-\Gamma$, then

$$
\vec{e}_{0}, \ldots, \vec{e}_{k-1}, \gamma_{+} \vec{e}_{0}, \ldots, \gamma_{+} \vec{e}_{k-1}
$$

is a sequence of consecutive oriented edges, and the even vertex of $\gamma_{+} \vec{v}_{k-1}$ is equal to $\gamma v_{0}$. Note that $\sum_{\sigma \in \Sigma} \iota P_{0}^{\sigma}$ is equal in $\mathbb{Z}\left[\overrightarrow{\mathcal{E}}\left(\mathcal{G}_{+}\right)\right]$to $\vec{e}_{0}+\vec{e}_{1}+\ldots+\vec{e}_{k-1}$ if $\iota=1$, and to

$$
\vec{e}_{0}+\vec{e}_{1}+\ldots+\vec{e}_{k-1}+\gamma_{+} \vec{e}_{0}+\gamma_{+} \vec{e}_{1}+\ldots+\gamma_{+} \vec{e}_{k-1}
$$

if $\iota=2$. Denote by $e_{i}$ the unoriented edge of $\mathcal{T}$ corresponding to $\vec{e}_{i}$, and let $w$ be any element of $\Gamma_{+}-\Gamma$. By definition of the bijection $\kappa$ of lemma 2.2, the following equalities hold in $\mathbb{Z}[\mathcal{E}(\mathcal{G})]$ :

$$
\begin{gathered}
\kappa^{-1}\left(\vec{e}_{0}+\ldots+\vec{e}_{k-1}\right)=e_{0}+w e_{1}+\ldots+e_{k-2}+w e_{k-1} \quad \text { if } \iota=1, \\
\left.\kappa^{-1}\left(\vec{e}_{0}+\ldots+\vec{e}_{k-1}+\gamma_{+} \vec{e}_{0}+\gamma_{+} \vec{e}_{1}+\ldots+\gamma_{+} \vec{e}_{k-1}\right)\right)=e_{0}+w e_{1}+\ldots \\
+e_{k-1}+w\left(\gamma_{+} e_{0}\right)+\left(\gamma_{+} e_{1}\right)+\ldots+w\left(\gamma_{+} e_{k-1}\right) \quad \text { if } \iota=2 .
\end{gathered}
$$

Projecting the right hand sides of the above equalities to $\mathbb{Z}[\mathcal{E}(\mathcal{G})]_{\mathrm{sp}}$, and keeping into account that $w$ acts as -1 on this module, gives in both cases $\operatorname{path}\left(v_{0}, \gamma v_{0}\right)$.

The next proposition elucidates the relation between the $p$-adic Shintani cycle defined above and the special values of the complex $L$-function of $E / K$. Following the notations of section 3 , fix Gross points $P_{0}=P_{0}^{1}, \ldots, P_{0}^{h}$ which are representatives for the $\Sigma$-orbits of the Gross points of conductor $c$, and list the elements of $\Delta$ so that $\left[\delta_{i} P_{0}^{1}\right]=\left[P_{0}^{i}\right]$, where $\left[P_{0}^{i}\right]$ denotes the $\Sigma$-orbit of $P_{0}^{i}$. As above, the Gross point $P_{0}^{i}$ determines a $p$-adic Shintani cycle $\mathfrak{c}_{i} \in \bar{\Gamma}$, with $\mathfrak{c}_{1}=\mathfrak{c}$. Given a complex character $\chi: \Delta \rightarrow \mathbb{C}^{\times}$of $\Delta$, set

$$
\begin{gathered}
\mathfrak{c}_{H}:=\sum_{i=1}^{h} \mathfrak{c}_{i} \otimes \delta_{i}^{-1} \in \bar{\Gamma} \otimes \mathbb{Z}[\Delta] \\
\mathfrak{c}_{K, \chi}:=\chi\left(\mathfrak{c}_{H}\right)=\sum_{i=1}^{h} \mathfrak{c}_{i} \otimes \chi\left(\delta_{i}\right)^{-1} \in \bar{\Gamma} \otimes \mathbb{Z}[\chi] .
\end{gathered}
$$

If $\chi$ is the trivial character, we will also write $\mathfrak{c}_{K}$ as a shorthand for $\mathfrak{c}_{K, \chi}$. Extend the pairing $\langle$,$\rangle on \bar{\Gamma}$ to a hermitian pairing on $\bar{\Gamma} \otimes \mathbb{Z}[\chi]$.

\section{Proposition 5.2}

Suppose that $\chi$ is primitive. The following equality holds:

$$
\left\langle\eta_{f} \mathfrak{c}_{K, \chi}, \mathfrak{c}_{K, \chi}\right\rangle=\frac{L(E / K, \chi, 1)}{\Omega_{f}} \sqrt{d} \cdot(\iota u)^{2} \cdot n_{f}
$$

Proof. In view of lemma 5.1, this is simply a restatement of the results of [Gr] and [Dag]. 
Recall the maps $j: \bar{\Gamma} \rightarrow \mathcal{N} \otimes \mathbb{Q}_{p}^{\times}$and $\eta_{f} \otimes \mathrm{id}: \mathcal{N} \otimes \mathbb{C}_{p}^{\times} \rightarrow \mathbb{C}_{p}^{\times}$defined in section 4. By an abuse of notation, we denote in the same way the maps obtained by extending scalars to $\mathbb{Z}[\chi]$.

\section{Corollary 5.3}

The equality

$$
\left(\eta_{f} \otimes \mathrm{id}\right)\left(j\left(\mathfrak{c}_{K, \chi}\right)\right)=q \otimes \rho
$$

holds in $\mathbb{Q}_{p}^{\times} \otimes \mathbb{Z}[\chi]$, where $\rho \in \mathbb{Z}[\chi]$ satisfies

$$
|\rho|^{2}=\frac{L(E / K, \chi, 1)}{\Omega_{f}} \sqrt{d} \cdot(\iota u)^{2} \cdot n_{f} .
$$

Proof. By proposition 4.4 combined with the definition of $\eta_{f}$ given in section $2, \rho$ is equal to $\left\langle\mathfrak{c}_{K, \chi}, e^{f}\right\rangle \in \mathbb{Z}[\chi]$. Hence

$$
\begin{aligned}
|\rho|^{2} & =\left\langle\mathfrak{c}_{K, \chi}, e^{f}\right\rangle\left\langle e^{f}, \mathfrak{c}_{K, \chi}\right\rangle \\
& =\left\langle\eta_{f} \mathfrak{c}_{K, \chi}, \mathfrak{c}_{K, \chi}\right\rangle .
\end{aligned}
$$

The claim follows from proposition 5.2.

\section{Remark 5.4}

Let $F$ be a real quadratic field and let $\psi: F \rightarrow M_{2}(\mathbb{Q})$ be an embedding. Assume that $\psi$ maps the ring of integers $\mathcal{O}_{F}$ to the Eichler order $M_{0}(N)$ of integral matrices with lower left entry divisible by $N$. Since the homology group $H_{1}\left(X_{0}(N), \mathbb{Z}\right)$ can be identified with the maximal torsion-free abelian quotient of $\Gamma_{0}(N)$, the image by $\psi$ of a fundamental unit in $\mathcal{O}_{F}$ of norm 1 determines an integral homology cycle $\mathfrak{s} \in H_{1}\left(X_{0}(N), \mathbb{Z}\right)$. Shintani $[\mathrm{Sh}]$ proved that the cycle $\mathfrak{s}$ encodes the special values of the classical $L$-series over $F$ attached to newforms on $X_{0}(N)$. In light of proposition 5.2 , the element $\mathfrak{c}$ can be viewed as a $p$-adic analogue of the cycle $\mathfrak{s}$.

\section{6 p-adic Shintani cycles and derivatives of p-adic L-functions}

Let $P_{0}$ be a Gross point of conductor $c$. In section 5 , we attached to $P_{0}$ a $p$-adic cycle $\mathfrak{c} \in \bar{\Gamma}$, and proved in proposition 5.2 that $\mathfrak{c}$ is related to the special values of the complex $L$-function of $E / K$. Our main result (theorem 6.1 below) shows that $\mathfrak{c}$ is also related to the first derivative of the $p$-adic $L$-function defined in section 3 . By combining these results we will obtain theorem 1.1.

Write $\underline{j}$ for the composite map

$$
\bar{\Gamma} \stackrel{j}{\longrightarrow} \mathcal{N} \otimes \mathbb{Q}_{p}^{\times} \rightarrow \mathcal{N}_{\mathrm{sp}} \otimes \mathbb{Q}_{p}^{\times} \rightarrow \mathcal{N}_{\mathrm{sp}} \otimes G_{\infty}
$$

where the second map is induced by the natural projection of $\mathcal{N}$ onto $\mathcal{N}_{\text {sp }}$, and the third map is induced by $\operatorname{rec}_{p}: \mathbb{Q}_{p}^{\times} \rightarrow G_{\infty}$. Our main result is the following.

\section{Theorem 6.1}

The following equality holds up to sign in $\mathcal{N}_{\mathrm{sp}} \otimes G_{\infty}$ :

$$
\mathcal{L}_{p}^{\prime}\left(\mathcal{N}_{\mathrm{sp}} / H, P_{0}\right)^{\iota}=\underline{j}(\mathfrak{c})
$$


Recall the definition of the elements $\mathfrak{c}_{H}$ and $\mathfrak{c}_{K}$ given in section 5. By lemma 3.8, we obtain directly:

\section{Corollary 6.2}

(i) The following equality holds up to sign in $\mathcal{N}_{\mathrm{sp}} \otimes \mathbb{Z}[\Delta] \otimes G_{\infty}$ :

$$
\mathcal{L}_{p}^{\prime}\left(\mathcal{N}_{\mathrm{sp}} / H\right)^{\iota}=\underline{j}\left(\mathfrak{c}_{H}\right) \text {. }
$$

(ii) The following equality holds up to sign in $\mathcal{N}_{\mathrm{sp}} \otimes G_{\infty}$ :

$$
\mathcal{L}_{p}^{\prime}\left(\mathcal{N}_{\mathrm{sp}} / K\right)^{\iota}=\underline{j}\left(\mathfrak{c}_{K}\right) \text {. }
$$

By applying the operator $\eta_{f}$ to both sides of the equalities of corollary 6.2 , and using corollary 4.5 and the definitions of the $p$-adic $L$-functions attached to $\mathcal{N}_{\mathrm{sp}}$ and $E$, we find:

\section{Corollary 6.3}

(i) The following equality holds up to sign in $\mathbb{Z}[\Delta] \otimes G_{\infty}$ :

$$
c_{p} \mathcal{L}_{p}^{\prime}(E / H)^{\iota}=\underline{j}\left(\eta_{f} \mathfrak{c}_{H}\right) .
$$

(ii) The following equality holds up to sign in $G_{\infty}$ :

$$
c_{p} \mathcal{L}_{p}^{\prime}(E / K)^{\iota}=\underline{j}\left(\eta_{f} \mathfrak{c}_{K}\right) .
$$

Proof of theorem 1.1

Combine corollary 6.3 with corollary 5.3.

By combining corollary 6.3 with corollary 5.3, we also obtain the following generalization of theorem 1.1. Let $\mathcal{L}_{p}^{\prime}(E / K, \chi)$ stand for the element $\chi\left(\mathcal{L}_{p}^{\prime}(E / H)\right)$ of $G_{\infty} \otimes \mathbb{Z}[\chi]$

\section{Theorem 6.4}

Suppose that $\chi$ is primitive. The following equalities hold up to sign:

$$
c_{p} \mathcal{L}_{p}^{\prime}(E / K, \chi)=\operatorname{rec}_{p}(q) \otimes \rho \quad \text { in } G_{\infty} \otimes \mathbb{Z}\left[\frac{1}{2}\right][\chi]
$$

and

$$
\mathcal{L}_{p}^{\prime}(E / K, \chi)=\frac{\operatorname{rec}_{p}(q)}{\operatorname{ord}_{p}(q)} \otimes \rho \quad \text { in } G_{\infty} \otimes \mathbb{Q}[\chi]
$$

where

$$
|\rho|^{2}=\frac{L(E / K, \chi, 1)}{\Omega_{f}} \cdot d^{\frac{1}{2}} u^{2} n_{f} .
$$




\section{Corollary 6.5}

The derivative $\mathcal{L}_{p}^{\prime}(E / K, \chi)$ is non-zero in $G_{\infty} \otimes \mathbb{Q}[\chi]$ if and only if the classical special value $L(E / K, \chi, 1)$ is non-zero.

Proof. By theorem 6.4, one is reduced to showing that $\operatorname{rec}_{p}(q)$ is a non-torsion element of $G_{\infty}$, i.e., $q^{p-1}$ does not belong to the kernel of the reciprocity map. But elements in this kernel are algebraic over $\mathbb{Q}$, and $q$ is known to be transcendental by a result of Barré-Sirieix, Diaz, Gramain and Philibert [BSDGP].

\section{Remark 6.6}

Theorem 1.1 was conjectured in [BD1], section 5.1 in a slightly different form. We conclude this section by studying the compatibility of theorem 1.1 (and its generalization theorem 6.4) with the conjectures of [BD1]. For simplicity, assume throughout this remark that the elliptic curve $E$ is semistable, so that $N$ is squarefree, and that $E$ is isolated in its isogeny class, so that the action of $\operatorname{Gal}(\overline{\mathbb{Q}} / \mathbb{Q})$ on the $\ell$-torsion points of $E$ is irreducible for all primes $\ell$.

Let $p_{1} \cdots p_{n} q_{1} \cdots q_{n}$ be a prime factorization of the squarefree integer $p N^{-}$, with $p_{1}=p$. Denote by $X_{1}$ the Shimura curve $X$, and by $X_{n+1}$ the classical modular curve $X_{0}(N)$. For $i=2, \ldots, n$, denote by $X_{i}$ the Shimura curve associated with an Eichler order of level $N^{+} p_{1} \cdots p_{i-1} q_{1} \cdots q_{i-1}$ in the indefinite quaternion algebra of discriminant $p_{i} \cdots p_{n} q_{i} \cdots q_{n}$. Since $E$ is modular, the Jacquet-Langlands correspondence [JL] implies that $E$ is parametrized by the jacobian $J_{i}$ of the curve $X_{i}$, $i=1, \ldots, n+1$. Let

$$
\phi_{i}: J_{i} \rightarrow E
$$

be the strong Weil parametrization of $E$ by $J_{i}$. Thus, the morphism $\phi_{i}$ has connected kernel, and its dual $\phi_{i}^{\vee}: E \rightarrow J_{i}$ is injective. The endomorphism $\phi_{i} \circ \phi_{i}^{\vee}$ of $E$ is multiplication by an integer $d_{X_{i}}$, called the degree of the modular parametrization of $E$ by the Shimura curve $X_{i}$.

If $\ell \mid N$, denote by $c_{\ell}$ the order of the group of connected components of $E$ at $\ell$.

\section{Theorem 6.7 (Ribet-Takahashi)}

Under our assumptions:

i)

$$
\frac{d_{X_{0}(N)}}{d_{X}}=c_{p_{1}} \cdots c_{p_{n}} c_{q_{1}} \cdots c_{q_{n}}
$$

ii)

$$
\left\langle e^{f}, e^{f}\right\rangle=d_{X} c_{p}
$$

Proof. Part i) follows from theorem 1 of $[\mathrm{RT}]$. Part ii) follows from section 2 of [RT]. The results of [RT] exclude the case where $N^{+}$is prime, but a forthcoming paper of S. Takahashi will deal with this case as well.

By combining theorem 6.7 with the relation $\Omega_{f}=d_{X_{0}(N)} \cdot \Omega_{E}$, where $\Omega_{E}$ is the complex period of $E$, we find that the formula of theorem 1.1 (and likewise for theorem 6.4) becomes

$$
\mathcal{L}_{p}^{\prime}(E / K)=\frac{\operatorname{rec}_{p}(q)}{\operatorname{ord}_{p}(q)} \sqrt{L(E / K, 1) \Omega_{E}^{-1} \cdot d^{\frac{1}{2}} u^{2} \prod_{\ell \mid N^{-}} c_{\ell}^{-1}},
$$


which is the same as conjecture 5.3 of [BD1].

\section{Proof of theorem 6.1}

First, we give an explicit description of certain group actions on the $p$-adic upper half plane and on the Bruhat-Tits tree depending on our choice of a Gross point $P_{0}$ of conductor $c$. Then, we compute the value $\underline{j}(\mathfrak{c})$, for $\mathfrak{c}$ as in sections 5 and 6 .

\section{Group actions on $\mathcal{H}_{p}$ and $\mathcal{T}$}

Let $K_{p}:=K \otimes \mathbb{Q}_{p}$. Our choice of a prime $\mathfrak{p}$ above $p$ determines an identification of $K_{p}=K_{\mathfrak{p}} \times K_{\overline{\mathfrak{p}}}$ with $\mathbb{Q}_{p} \times \mathbb{Q}_{p}$.

As in section 5 , choose a representative $\left(R_{0}, \xi_{0}\right)$ for the Gross point $P_{0}$ such that $R_{0}\left[\frac{1}{p}\right]$ and $R\left[\frac{1}{p}\right]$ are equal. Let $\left(\vec{e}_{0}, \xi_{0}\right)$ be a pair corresponding to $P_{0}$, and denote by $v_{0}$ the origin of $\vec{e}_{0}$. Set $R_{0, p}:=R_{0} \otimes \mathbb{Z}_{p}$, and let $\underline{R}_{0, p}$ be the maximal order of $B_{p}$ corresponding to $v_{0}$. Recall the isomorphism

$$
\psi: B_{p} \rightarrow M_{2}\left(\mathbb{Q}_{p}\right)
$$

fixed in section 4 . We may, and will from now on, choose $\psi$ so that:

i) $\psi$ maps $\underline{R}_{0, p}$ onto $M_{2}\left(\mathbb{Z}_{p}\right)$;

ii) $\psi \circ \xi_{0} \operatorname{maps}(x, y) \in K_{p}=\mathbb{Q}_{p} \times \mathbb{Q}_{p}$ to the diagonal matrix $\left(\begin{array}{ll}x & 0 \\ 0 & y\end{array}\right)$.

Condition i) allows us to identify $\mathcal{T}=\mathbb{Q}_{p}^{\times} \underline{R}_{0, p}^{\times} \backslash B_{p}^{\times}$with $\operatorname{PGL}_{2}\left(\mathbb{Z}_{p}\right) \backslash \mathrm{PGL}_{2}\left(\mathbb{Q}_{p}\right)$. Viewing $K_{p}^{\times}$as a subgroup of $\mathrm{GL}_{2}\left(\mathbb{Q}_{p}\right)$ thanks to the embedding $\psi \circ \xi_{0}$ yields actions of $K_{p}^{\times}$on $\mathcal{H}_{p}$ and on $\mathcal{T}=\mathrm{PGL}_{2}\left(\mathbb{Z}_{p}\right) \backslash \mathrm{PGL}_{2}\left(\mathbb{Q}_{p}\right)$, factoring through $K_{p}^{\times} / \mathbb{Q}_{p}^{\times}$. Identify this last group with $\mathbb{Q}_{p}^{\times}$by mapping a pair $(x, y)$ modulo $\mathbb{Q}_{p}^{\times}$to $x y^{-1}$. Under this identification, an element $x$ of $\mathbb{Q}_{p}^{\times}$acts on $\mathcal{H}_{p}$ as multiplication by $x$, and on $\mathcal{T}$ as conjugation by the matrix $\left(\begin{array}{ll}x & 0 \\ 0 & 1\end{array}\right)$.

Recall the element $v \in \mathcal{O} \subset K_{p}^{\times}$defined in section 5 by $\mathfrak{p}^{k}=(v)$. Identify as above $v$ with an element $\underline{w}$ of $\mathbb{Q}_{p}^{\times}$. Note that $\underline{w}$ is equal to $p^{k}$ times a $p$-adic unit. Set $\tilde{G}_{\infty}:=\mathbb{Q}_{p}^{\times}=p^{\mathbb{Z}} \times \mathbb{Z}_{p}^{\times}$. Define the quotients of $\tilde{G}_{\infty}$

$$
\tilde{\Sigma}:=\mathbb{Q}_{p}^{\times} / \mathbb{Z}_{p}^{\times}=p^{\mathbb{Z}}, \quad \tilde{G}_{n}:=p^{\mathbb{Z}} \times\left(\mathbb{Z}_{p} / p^{n} \mathbb{Z}_{p}\right)^{\times}, n \geq 1 .
$$

To simplify slightly the computation, assume from now on that $\mathcal{O}^{\times}=\{ \pm 1\}$. (If $\mathcal{O}^{\times} \neq\{ \pm 1\}$, then $K$ has discriminant -3 or -4 , and the exact sequences below have to be modified to account for the non-trivial units of $\mathcal{O}$. The computations in this case follow closely those presented in the paper.) Class field theory yields the exact sequence

$$
0 \rightarrow\langle\underline{w}\rangle \rightarrow \tilde{G}_{\infty} \stackrel{\mathrm{rec}_{p}}{\longrightarrow} G_{\infty} \rightarrow 0
$$

and the induced sequences

$$
0 \rightarrow\langle\underline{w}\rangle \rightarrow \tilde{\Sigma} \rightarrow \Sigma \rightarrow 0, \quad 0 \rightarrow\langle\underline{w}\rangle \rightarrow \tilde{G}_{n} \rightarrow G_{n} \rightarrow 0 .
$$

For $n \geq 0$, denote by $\mathbb{Z}_{p}^{(n)} \subset \tilde{G}_{\infty}$ the subgroup of elements of $\mathbb{Z}_{p}^{\times}$which are congruent to 1 modulo $p^{n}$. 
Definition. We say that a vertex $v$ of $\mathcal{T}$ has level $n$, and write $\ell(v)=n$, if the stabilizer of $v$ for the action of $\tilde{G}_{\infty}$ is equal to $\mathbb{Z}_{p}^{(n)}$. Likewise, we say that an edge $e$ of $\mathcal{T}$ has level $n$, and write $\ell(e)=n$, if the stabilizer of $e$ for the action of $\tilde{G}_{\infty}$ is $\mathbb{Z}_{p}^{(n)}$

Note that the group $\tilde{G}_{n}(\tilde{\Sigma}$ if $n=0)$ acts simply transitively on the vertices and edges of level $n$. By definition of the action of $\tilde{G}_{\infty}$ on $\mathcal{T}, v_{0}$ is a vertex of level 0 . Thus, the set of vertices of level 0 is equal to the $\tilde{\Sigma}$-orbit of $v_{0}$. More generally, the set of vertices of level $n$ can be described as the $\tilde{G}_{n}$-orbit of a vertex $v_{n}$, whose distance from $v_{0}$ is $n$ and whose distance from all the other vertices in the orbit $\tilde{\Delta} v_{0}$ is $>n$.

By using the standard coordinate, identify $\mathbb{P}^{1}\left(\mathbb{C}_{p}\right)$ with $\mathbb{C}_{p} \cup\{\infty\}$ and $\mathcal{H}_{p}$ with $\mathbb{P}^{1}\left(\mathbb{C}_{p}\right)-\mathbb{P}^{1}\left(\mathbb{Q}_{p}\right)$. In particular, view 0 and $\infty$ as elements of $\mathbb{P}^{1}\left(\mathbb{Q}_{p}\right)$. Recall the element $\gamma=\gamma\left(P_{0}\right)$ of $\Gamma$ defined in section 5. Since the reduced norm of $\gamma$ has positive valuation, our choice of the isomorphism $\psi$ yields

$$
\lim _{n \rightarrow+\infty} \gamma^{n} z=0, \quad \lim _{n \rightarrow-\infty} \gamma^{n} z=\infty
$$

for all $z \in \mathcal{H}_{p}$. Note also that 0 and $\infty$ are the fixed points for the action of $\tilde{G}_{\infty}$ on $\mathbb{P}^{1}\left(\mathbb{C}_{p}\right)$.

Let $\mathcal{H}_{p}\left(\mathbb{Q}_{p^{2}}\right)=\mathbb{Q}_{p^{2}}-\mathbb{Q}_{p}$ be the $\mathbb{Q}_{p^{2}}$-points of the $p$-adic upper half plane. Define the reduction map

$$
r: \mathcal{H}_{p}\left(\mathbb{Q}_{p^{2}}\right) \rightarrow \mathcal{V}(\mathcal{T})
$$

as follows. Given $z \in \mathcal{H}_{p}\left(\mathbb{Q}_{p^{2}}\right)$, let $\mathcal{Q}_{z}$ denote the stabilizer of $z$ in $\operatorname{GL}_{2}\left(\mathbb{Q}_{p}\right)$, together with the zero matrix. Then $\mathcal{Q}_{z}$ is a field isomorphic to $\mathbb{Q}_{p^{2}}$, and this gives rise to an embedding of $\mathbb{Q}_{p^{2}}$ in $M_{2}\left(\mathbb{Q}_{p}\right)$ (well-defined up to an isomorphism of $\mathbb{Q}_{p^{2}}$ ). Write $\mathbb{Z}_{p^{2}}$ for the ring of integers of $\mathbb{Q}_{p^{2}}$, and let $S$ be the unique maximal order of $M_{2}\left(\mathbb{Q}_{p}\right)$ containing the image of $\mathbb{Z}_{p^{2}}$ by the above embedding. We have $r(z)=S$. (See also [BD2], section 1.)

\section{Lemma 7.1}

1) The reduction map $r$ is $\mathrm{GL}_{2}\left(\mathbb{Q}_{p}\right)$-equivariant. In particular, $r$ is equivariant for the group actions defined above.

2) Write $\mathbb{Z}_{p^{2}}=\mathbb{Z}_{p} \alpha+\mathbb{Z}_{p}$. We have $r^{-1}\left(v_{0}\right)=\mathbb{Z}_{p}^{\times} \alpha+\mathbb{Z}_{p}$.

3) If $z_{1}$ and $z_{2}$ are mapped by $r$ to adjacent vertices of respective levels $n$ and $n+1$, then $z_{1} z_{2}^{-1} \equiv 1\left(\bmod p^{n}\right)$.

Proof.

1) Let $z$ be an element of $\mathcal{H}_{p}\left(\mathbb{Q}_{p^{2}}\right)$, and let $B$ be a matrix in $\mathrm{GL}_{2}\left(\mathbb{Q}_{p}\right)$. If $f$ : $\mathbb{Q}_{p^{2}} \rightarrow M_{2}\left(\mathbb{Q}_{p}\right)$ is an embedding fixing $z$, then $B f B^{-1}$ is an embedding fixing $B z$. Suppose that $S$ is the maximal ideal containing $f\left(\mathbb{Z}_{p^{2}}\right)$. Then $B S B^{-1}=S * B^{-1}$ is the maximal ideal containing the image of $\mathbb{Z}_{p^{2}}$ by $B f B^{-1}$. Thus, $r(B z)=S * B^{-1}$, as was to be shown.

2) Suppose to fix ideas that $p>2$. Then, we may assume that $\alpha=\sqrt{\nu}$, where the integer $\nu$ is not a squaremodulo $p$. (The case $p=2$ can be dealt with in a similar way, for instance by taking $\alpha=(1+\sqrt{-3}) / 2$.) A direct computation shows that

$$
\mathcal{Q}_{\sqrt{\nu}}=\left\{\left(\begin{array}{cc}
b & a \nu \\
a & b
\end{array}\right): a, b \in \mathbb{Q}_{p}\right\} .
$$


Mapping the above matrix to $a \sqrt{\nu}+b$ yields an isomorphism of $\mathcal{Q}_{\sqrt{\nu}}$ onto $\mathbb{Q}_{p^{2}}$. Thus, $r(\sqrt{\nu})$ is equal to $v_{0}=M_{2}\left(\mathbb{Z}_{p}\right)$. Given $z=a \sqrt{\nu}+b \in \mathcal{H}_{p}\left(\mathbb{Q}_{p^{2}}\right)$, we have $z=B \sqrt{\nu}$, where $B$ is the matrix $\left(\begin{array}{ll}a & b \\ 0 & 1\end{array}\right)$. By part 1 ,

$$
r(z)=B M_{2}\left(\mathbb{Z}_{p}\right) B^{-1} .
$$

But $B M_{2}\left(\mathbb{Z}_{p}\right) B^{-1}=M_{2}\left(\mathbb{Z}_{p}\right)$ if and only if $B$ belongs to $\mathrm{GL}_{2}\left(\mathbb{Z}_{p}\right)$, i.e., $a$ belongs to $\mathbb{Z}_{p}^{\times}$.

3) Set $r\left(z_{1}\right)=v_{1}$ and $r\left(z_{2}\right)=v_{2}$. The edge joining $v_{1}$ to $v_{2}$ has level $n+1$. Since $\tilde{G}_{\infty}=\mathbb{Q}_{p}^{\times}$acts transitively on the edges of level $n+1$, there is $g \in \mathbb{Q}_{p}^{\times}$such that $g v_{1}$ and $g v_{2}$ have distance from $v_{0}$ equal to $n$ and $n+1$, respectively. With notations as in the proof of part 2 of this proposition, write $g z_{i}=a_{i} \sqrt{\nu}+b_{i}, i=1,2$, where $a_{i}, b_{i} \in \mathbb{Z}_{p}, \operatorname{gcd}\left(a_{i}, b_{i}\right)=1$, and $p^{n}\left\|a_{1}, p^{n+1}\right\| a_{2}$. Thus, the vertex $g v_{i}$ is represented by the matrix

$$
A_{i}=\left(\begin{array}{cc}
a_{i} & b_{i} \\
0 & 1
\end{array}\right)
$$

Our assumption on $g v_{1}$ and $g v_{2}$ implies that the column $\left(\begin{array}{c}b_{2} \\ 1\end{array}\right)$ of $A_{2}$ is a $\mathbb{Z}_{p}$-linear combination of the columns of $A_{1}$. It follows that $b_{1} \equiv b_{2}\left(\bmod p^{n}\right)$, and hence

$$
z_{1} z_{2}^{-1}=g z_{1}\left(g z_{2}\right)^{-1} \equiv 1 \quad\left(\bmod p^{n}\right) .
$$

\section{The calculation}

Given $\delta \in \Gamma$, write as usual $\bar{\delta}$ for the natural image of $\delta$ in $\bar{\Gamma}$. We now compute explicitly the value of $j(\mathfrak{c})(\bar{\delta})=[\mathfrak{c}, \bar{\delta}]$, for $\delta \in \Gamma$. We begin with the following lemma.

\section{Lemma 7.2}

Given $\delta \in \Gamma$, we have

$$
j(\mathfrak{c})(\bar{\delta})=\prod_{\epsilon \in \mathcal{S}} \frac{\epsilon \delta z_{0}}{\epsilon z_{0}},
$$

where $z_{0}$ is any element in $\mathcal{H}_{p}$, and $\mathcal{S}$ is any set of representatives for $\langle\gamma\rangle \backslash \Gamma$.

Proof. (Cf. [M], theorem 2.8.)

Let $\mathcal{S}^{\prime}$ be any set of representatives for $\Gamma /\langle\gamma\rangle$. In view of the formulae (4), for any $z_{0}$ and $a$ in $\mathcal{H}_{p}$ we have the chain of equalities

$$
\begin{aligned}
j(\mathfrak{c})(\bar{\delta}) & =\prod_{\epsilon \in \Gamma} \frac{z_{0}-\epsilon a}{z_{0}-\epsilon \gamma a} \cdot \frac{\delta z_{0}-\epsilon \gamma a}{\delta z_{0}-\epsilon a} \\
& =\prod_{\epsilon \in \mathcal{S}^{\prime}} \prod_{n=-\infty}^{+\infty} \frac{z_{0}-\epsilon \gamma^{n} a}{z_{0}-\epsilon \gamma^{n+1} a} \cdot \frac{\delta z_{0}-\epsilon \gamma^{n+1} a}{\delta z_{0}-\epsilon \gamma^{n} a} \\
& =\prod_{\epsilon \in \mathcal{S}^{\prime}} \lim _{N \rightarrow+\infty} \frac{z_{0}-\epsilon \gamma^{-N} a}{z_{0}-\epsilon \gamma^{N+1} a} \cdot \frac{\delta z_{0}-\epsilon \gamma^{N+1} a}{\delta z_{0}-\epsilon \gamma^{-N} a} \\
& =\prod_{\epsilon \in \mathcal{S}^{\prime}} \frac{z_{0}-\epsilon \infty}{z_{0}-\epsilon 0} \cdot \frac{\delta z_{0}-\epsilon 0}{\delta z_{0}-\epsilon \infty} \\
& =\prod_{\epsilon \in \mathcal{S}^{\prime}} \frac{\epsilon^{-1} \delta z_{0}}{\epsilon^{-1} z_{0}} .
\end{aligned}
$$


Note that $\left(\mathcal{S}^{\prime}\right)^{-1}$ is a set of representatives for $\langle\gamma\rangle \backslash \Gamma$, and any set of representatives for $\langle\gamma\rangle \backslash \Gamma$ can be obtained in this way. The claim follows.

\section{Lemma 7.3}

Let $d$ be an edge of $\mathcal{T}$, let $n$ be a positive integer, and let $\mathcal{S}$ be a set of representatives for $\langle\gamma\rangle \backslash \Gamma$. Then the set $\{\epsilon \in \mathcal{S}: \ell(\epsilon d) \leq n\}$ is finite.

Proof. If $\left\{\epsilon_{i}\right\}$ is a sequence of distinct elements of $\mathcal{S}$ such that $\ell\left(\epsilon_{i} d\right) \leq n$, we can find integers $k_{i}$ such that $\gamma^{k_{i}} \epsilon_{i} d$ describes only finitely many edges. This contradicts the discreteness of $\Gamma$.

We say that two elements of $\bar{\Gamma}$ are linearly independent if they generate a rank two free abelian subgroup of $\bar{\Gamma}$.

\section{Proposition 7.4}

1) Suppose that $\mathfrak{c}$ and $\bar{\delta}$ are linearly independent in $\bar{\Gamma}$. There exists a set $\mathcal{S}$ of representatives for $\langle\gamma\rangle \backslash \Gamma$ such that if $\epsilon$ belongs to $\mathcal{S}$, then all the elements of the coset $\epsilon\langle\delta\rangle$ belong to $\mathcal{S}$.

2) There exists a set $\mathcal{S}=\mathcal{S}_{0} \amalg \mathcal{S}_{1}$ of representatives for $\langle\gamma\rangle \backslash \Gamma$ such that:

(i) the set $\mathcal{S}_{0}$ contains a finite number of elements which are mapped by the isomorphism $\psi$ to diagonal matrices of $\mathrm{PGL}_{2}\left(\mathbb{Q}_{p}\right)$;

(ii) if $\epsilon$ belongs to $\mathcal{S}_{1}$, then all the elements of the coset $\epsilon\langle\gamma\rangle$ belong to $\mathcal{S}_{1}$.

Proof. (Cf. [M], lemma 2.7)

1) Consider a decomposition of $\Gamma$ as disjoint union of double cosets

$$
\Gamma=\coprod_{\bar{\epsilon} \in \overline{\mathcal{S}}}\langle\gamma\rangle \bar{\epsilon}\langle\delta\rangle .
$$

We claim that we may take $\mathcal{S}$ to be $\left\{\bar{\epsilon} \delta^{m}: \bar{\epsilon} \in \overline{\mathcal{S}}, m \in \mathbb{Z}\right\}$. For, if $\bar{\epsilon} \delta^{m}=\gamma^{r} \bar{\epsilon} \delta^{n}$, we find $\delta^{m-n}=\bar{\epsilon}^{-1} \gamma^{r} \bar{\epsilon}$. Projecting this relation to $\bar{\Gamma}$ gives $m=n$.

2) Consider a decomposition of $\Gamma$ as disjoint union of double cosets

$$
\Gamma=\coprod_{\bar{\epsilon} \in \overline{\mathcal{S}}}\langle\gamma\rangle \bar{\epsilon}\langle\gamma\rangle
$$

Define $\mathcal{S}_{1}$ to be the set of elements of $\Gamma$ of the form $\bar{\epsilon} \gamma^{m}, m \in \mathbb{Z}$, where $\bar{\epsilon} \in \overline{\mathcal{S}}$ is such that $\langle\gamma\rangle \bar{\epsilon} \gamma^{n} \neq\langle\gamma\rangle \bar{\epsilon} \gamma^{m}$ whenever $m \neq n$. As for $\mathcal{S}_{0}$, we claim that it can be taken to be the set of elements $\bar{\epsilon} \in \overline{\mathcal{S}}$ which do not satisfy the above condition. In such a case, there is a relation $\gamma^{r} \bar{\epsilon} \gamma^{n}=\bar{\epsilon} \gamma^{m}$ for integers $r$ and $m \neq n$. Then, $\gamma^{r}=\bar{\epsilon} \gamma^{m-n} \bar{\epsilon}^{-1}$. By projecting this equality to $\bar{\Gamma}$, we see that $m-n=r$, and hence $\bar{\epsilon}$ and $\gamma^{r}$ commute. Since $\gamma^{r}$ is mapped by $\psi$ to the diagonal matrix $\left(\begin{array}{cc}\underline{w}^{\iota r} & 0 \\ 0 & 1\end{array}\right)$, where $\operatorname{ord}_{p}(\underline{w})=k>0$, a direct computation shows that $\bar{\epsilon}$ is also diagonal (and thus commutes with $\gamma$ ). Now consider the group of all the diagonal matrices in $\psi(\Gamma)$. Since $\Gamma$ is discrete, this group is the product of a finite group by a cyclic group containing the group generated by $\gamma$. In conclusion, the set $\mathcal{S}_{0}$ is finite, and

$$
\coprod_{\bar{\epsilon} \in \mathcal{S}_{0}}\langle\gamma\rangle \bar{\epsilon}\langle\gamma\rangle=\coprod_{\bar{\epsilon} \in \mathcal{S}_{0}}\langle\gamma\rangle \bar{\epsilon} .
$$


The claim follows.

In the computation of $\underline{j}(\mathfrak{c})(\bar{\delta})$, we can assume that either

(I) $\mathfrak{c}$ and $\bar{\delta}$ are linearly independent, or

(II) $\bar{\delta}=\mathfrak{c}$.

(In fact, if the rank of $\bar{\Gamma}$ is $>1$, it is enough to consider elements as in the first case, since the linear map $j(\mathfrak{c})$ is completely determined by the values $j(\mathfrak{c})(\bar{\delta})$, for $\mathfrak{c}$ and $\bar{\delta}$ linearly independent.) In the case (I), we use the notation $\mathcal{S}_{1}:=\mathcal{S}$, and the symbol $\mathcal{S}_{1}$ will always refer to a choice of representatives for $\langle\gamma\rangle \backslash \Gamma$ as in part 1 of proposition 7.4. In the case (II), the symbol $\mathcal{S}=\mathcal{S}_{0} \amalg \mathcal{S}_{1}$ will stand for a choice of representatives as in part 2 of proposition 7.4 .

\section{Lemma 7.5}

Let $\delta \in \Gamma$ be as in case (I) or (II) above. Then, the images in $G_{\infty}$ by the reciprocity map of $j(\mathfrak{c})(\bar{\delta})$ and $\prod_{\epsilon \in \mathcal{S}_{1}} \epsilon \delta z_{0} / \epsilon z_{0}$ are equal.

Proof. In the case (I) there is nothing to prove. In the case (II), proposition 7.4 combined with a direct computation shows that

$$
\prod_{\epsilon \in \mathcal{S}_{0}} \frac{\epsilon \gamma z_{0}}{\epsilon z_{0}}=\underline{w}^{\iota \#\left(\mathcal{S}_{0}\right)}
$$

Since $\underline{w}$ is in the kernel of the reciprocity map, the claim follows.

By lemma 7.5, we are now reduced to compute the product $\prod_{\epsilon \in \mathcal{S}_{1}} \epsilon \delta z_{0} / \epsilon z_{0}$, with $\delta$ as in case (I) or (II).

We begin with some preliminary remarks. Fix an edge $e$ of level equal to an odd integer $n$, having $v$ as its vertex of level $n$. Moreover, assume that the distance of $v$ from $v_{0}$ is also equal to $n$. Note that the image in $\mathcal{M}$ of $e$ is equal to the image in $\mathcal{M}$ of a Gross point of conductor $c p^{n}$.

Given $\tilde{\sigma} \in \tilde{G}_{n}$, define $\mu_{\tilde{\sigma}}$ to be equal to 1 , resp. -1 if $\tilde{\sigma} v$ has odd, resp. even distance from $v_{0}$. If $\iota=1$, observe that $\mu_{\tilde{\sigma}}$ depends only on the image $\bar{\sigma}$ of $\tilde{\sigma}$ in $\Sigma$ under the projection induced by the reciprocity map; in this case, we write $\mu_{\bar{\sigma}}$ instead of $\mu_{\tilde{\sigma}}$. If $\iota=2, \mu_{\tilde{\sigma}}$ is constant on the elements $\tilde{\sigma}$ which have the same image in $\Sigma$ and $p$-adic valuation of the same parity; moreover, the values of $\mu_{\tilde{\sigma}}$ corresponding to different parities are opposite. In this case, if $\tilde{\sigma}$ projects in $\Sigma$ to $\bar{\sigma}$ and $\operatorname{ord}_{p}(\tilde{\sigma})$ is even, we let $\mu_{\bar{\sigma}}$ stand for $\mu_{\tilde{\sigma}}$.

Given an edge $d$ of $\mathcal{T}$, and $\tilde{\sigma} \in \tilde{G}_{n}$, write $\tilde{\sigma} e \equiv d$ if the edge $\tilde{\sigma} e$ is $\mathcal{S}_{1}$-equivalent to $d$, and $\sigma e \approx d$ if the element $\sigma e$ of $\mathcal{M}$ is $\Gamma$-equivalent to $d$. If $\iota=1$, the relation $\tilde{\sigma} e \equiv d$ implies that $\sigma e \approx d$. If $\iota=2, \tilde{\sigma} e \equiv d$ yields $\sigma e \approx d$ when $\operatorname{ord}_{p}(\tilde{\sigma})$ is even, and $\sigma e \approx w d$, with $w \in \Gamma_{+}-\Gamma$, when $\operatorname{ord}_{p}(\tilde{\sigma})$ is odd.

Recall that $\omega_{d}$ denotes the order of the stabilizer in $\Gamma$ of $d$.

\section{Lemma 7.6}

1) Suppose that $\iota=1$. If the odd integer $n$ is sufficiently large, the projection $\tilde{G}_{n} \rightarrow G_{n}$ induces a $\omega_{d}$-to-1 map

$$
\left\{\tilde{\sigma} \in \tilde{G}_{n}: \tilde{\sigma} e \equiv d\right\} \rightarrow\left\{\sigma \in G_{n}: \sigma e \approx d\right\}
$$


2) Suppose that $\iota=2$. If the odd integer $n$ is sufficiently large, the projection $\tilde{G}_{n} \rightarrow G_{n}$ induces $\omega_{d}$-to-1 maps

$$
\left\{\tilde{\sigma} \in \tilde{G}_{n}: \tilde{\sigma} e \equiv d, \operatorname{ord}_{p}(\tilde{\sigma}) \text { even }\right\} \rightarrow\left\{\sigma \in G_{n}: \sigma e \approx d\right\}
$$

and

$$
\left\{\tilde{\sigma} \in \tilde{G}_{n}: \tilde{\sigma} e \equiv d, \operatorname{ord}_{p}(\tilde{\sigma}) \text { odd }\right\} \rightarrow\left\{\sigma \in G_{n}: \sigma e \approx w d\right\}
$$

Proof.

1) Suppose that $\tilde{\sigma}_{1} e \equiv d$ and $\tilde{\sigma}_{2} e \equiv d$, i.e., $\tilde{\sigma}_{1} e=\epsilon_{1} d$ and $\tilde{\sigma}_{2} e=\epsilon_{2} d$, for $\epsilon_{1}$ and $\epsilon_{2}$ in $\mathcal{S}_{1}$. If $\tilde{\sigma}_{1}$ and $\tilde{\sigma}_{2}$ have the same image in $G_{n}$, then $\tilde{\sigma}_{1}=\underline{w}^{r} \tilde{\sigma}_{2}$ for $r \in \mathbb{Z}$, and hence $\gamma^{r} \epsilon_{2} d=\epsilon_{1} d$. If $r \neq 0$, i.e., $\tilde{\sigma}_{1} \neq \tilde{\sigma}_{2}$ and $\epsilon_{1} \neq \epsilon_{2}$, then $\gamma^{r} \epsilon_{2} \epsilon_{1}^{-1}$ is a non-trivial element of the stabilizer in $\Gamma$ of $\epsilon_{1} d$, which is a group of cardinality $\omega_{d}$. Conversely, if $\tilde{\sigma}_{1} e=\epsilon_{1} d$ for $\epsilon_{1} \in \mathcal{S}_{1}$ and if $\beta$ is a non-trivial element of the stabilizer of $\epsilon_{1} d$, we have $\tilde{\sigma}_{1} e=\beta \epsilon_{1} d$. Write $\beta \epsilon_{1}=\gamma^{r} \epsilon_{2}, r \in \mathbb{Z}, \epsilon_{2} \in \mathcal{S}$. Then $\epsilon_{1} \neq \epsilon_{2}$. Note that if $n$ is large, then $\epsilon_{2}$ belongs to $\mathcal{S}_{1}$. We obtain $\underline{w}^{-r} \tilde{\sigma}_{1} e=\epsilon_{2} d$. This concludes the proof of part 1.

2) The proof is exactly the same as that of part 1.

Let

$$
\operatorname{path}\left(v_{0}, \delta v_{0}\right)=d_{1}-d_{2}+\cdots+d_{s-1}-d_{s} \in \mathbb{Z}[\mathcal{E}(\mathcal{T})]
$$

(Note that $s$ is even, since $\delta$ belongs to $\Gamma$.) Write $d_{j}=\left\{v_{j}^{e}, v_{j}^{o}\right\}$, where $v_{j}^{e}$ is the even vertex of $d_{j}$, and $v_{j}^{o}$ is the odd vertex of $d_{j}$. Note that we have

$$
\begin{aligned}
& v_{j}^{o}=v_{j+1}^{o} \quad \text { for } \quad j=1,3, \ldots, s-1, \\
& v_{j}^{e}=v_{j+1}^{e} \quad \text { for } \quad j=2,4, \ldots, s-2, \\
& v_{s}^{e}=\delta v_{1}^{e} .
\end{aligned}
$$

Fix $z_{0} \in \mathcal{H}_{p}\left(\mathbb{Q}_{p^{2}}\right)$ such that $r\left(z_{0}\right)=v_{0}$. We may choose elements $z_{j}^{o}$ and $z_{j}^{e}$ in $\mathcal{H}_{p}\left(\mathbb{Q}_{p^{2}}\right)$ such that $r\left(z_{j}^{o}\right)=v_{j}^{o}, r\left(z_{j}^{e}\right)=v_{j}^{e}$, and

$$
\begin{aligned}
& z_{j}^{o}=z_{j+1}^{o} \quad \text { for } \quad j=1,3, \ldots, s-1, \\
& z_{j}^{e}=z_{j+1}^{e} \quad \text { for } \quad j=2,4, \ldots, s-2, \\
& z_{1}^{e}=z_{0}, \quad z_{s}^{e}=\delta z_{0} .
\end{aligned}
$$

Hence

$$
\left(\epsilon z_{1}^{o}\right)\left(\epsilon z_{2}^{o}\right)^{-1} \cdots\left(\epsilon z_{s-1}^{o}\right)\left(\epsilon z_{s}^{o}\right)^{-1}=1, \quad\left(\epsilon z_{2}^{e}\right)\left(\epsilon z_{3}^{e}\right)^{-1} \cdots\left(\epsilon z_{s-2}^{e}\right)\left(\epsilon z_{s-1}^{e}\right)^{-1}=1,
$$

so that

$$
\prod_{\epsilon \in \mathcal{S}_{1}} \frac{\epsilon \delta z_{0}}{\epsilon z_{0}}=\prod_{\epsilon \in \mathcal{S}_{1}}\left(\frac{\epsilon z_{1}^{o}}{\epsilon z_{1}^{e}}\right)\left(\frac{\epsilon z_{2}^{o}}{\epsilon z_{2}^{e}}\right)^{-1} \cdots\left(\frac{\epsilon z_{s}^{o}}{\epsilon z_{s}^{e}}\right)^{-1}
$$

Fix a large odd integer $n$. For each $1 \leq j \leq s$, let $\mathcal{S}(j)$ be the set of elements $\epsilon$ in $\mathcal{S}_{1}$ such that $\epsilon d_{j}$ has level $\leq n$. Lemma 7.3 shows that the sets $\mathcal{S}(j)$ are finite. By lemma 7.1, we have the congruence

$$
\prod_{\epsilon \in \mathcal{S}_{1}} \frac{\epsilon \delta z_{0}}{\epsilon z_{0}} \equiv \prod_{\epsilon \in \mathcal{S}(1)}\left(\frac{\epsilon z_{1}^{o}}{\epsilon z_{1}^{e}}\right) \prod_{\epsilon \in \mathcal{S}(2)}\left(\frac{\epsilon z_{2}^{o}}{\epsilon z_{2}^{e}}\right)^{-1} \cdots \prod_{\epsilon \in \mathcal{S}(s)}\left(\frac{\epsilon z_{s}^{o}}{\epsilon z_{s}^{e}}\right)^{-1}\left(\bmod p^{n}\right)
$$


Each of the factors in the right hand side of equation (5) can be broken up into three contributions:

$$
\prod_{\mathcal{S}(j)} \frac{\epsilon z_{j}^{o}}{\epsilon z_{j}^{e}}=\prod_{\ell\left(\epsilon v_{j}^{o}\right)<n} \epsilon z_{j}^{o} \cdot \prod_{\ell\left(\epsilon v_{j}^{e}\right)<n}\left(\epsilon z_{j}^{e}\right)^{-1} \cdot \prod_{\ell\left(\epsilon d_{j}\right)=n}\left(\epsilon z_{j}^{\pi_{j}}\right)^{\mu_{j}}
$$

where $\pi_{j}=o$, resp. $\pi_{j}=e$ if the distance of the furthest vertex of $\epsilon d_{j}$ from $v_{0}$ is odd, resp. even, and where we set $\mu_{j}=1$ in the first case and $\mu_{j}=-1$ in the second case. By our choice of the set $\mathcal{S}_{1}$ as in proposition 7.4, the first two factors in this last expression cancel out in the formula (5). Hence we obtain

$$
\prod_{\epsilon \in \mathcal{S}_{1}} \frac{\epsilon \delta z_{0}}{\epsilon z_{0}} \equiv \prod_{\ell\left(\epsilon d_{1}\right)=n}\left(\epsilon z_{1}^{\pi_{1}}\right)^{\mu_{1}} \cdot \prod_{\ell\left(\epsilon d_{2}\right)=n}\left(\epsilon z_{2}^{\pi_{2}}\right)^{-\mu_{2}} \cdots \prod_{\ell\left(\epsilon d_{s}\right)=n}\left(\epsilon z_{s}^{\pi_{s}}\right)^{-\mu_{s}}\left(\bmod p^{n}\right) .
$$

As in the remarks before lemma 7.6, let $e$ be an edge of level $n$, such that its vertex $v$ of level $n$ has distance from $v_{0}$ also equal to $n$. Choose any $z \in \mathcal{H}_{p}\left(\mathbb{Q}_{p^{2}}\right)$ with $r(z)=v$. Since $\tilde{G}_{n}$ acts simply transitively on the set of edges of level $n$, lemma 7.1 gives

$$
\prod_{\epsilon \in \mathcal{S}_{1}} \frac{\epsilon \delta z_{0}}{\epsilon z_{0}} \equiv \prod_{\tilde{\sigma} e \equiv d_{1}}(\tilde{\sigma} z)^{\mu_{\tilde{\sigma}}} \cdot \prod_{\tilde{\sigma} e \equiv d_{2}}(\tilde{\sigma} z)^{-\mu_{\tilde{\sigma}}} \cdots \prod_{\tilde{\sigma} e \equiv d_{s}}(\tilde{\sigma} z)^{-\mu_{\tilde{\sigma}}}\left(\bmod p^{n}\right) .
$$

By lemma 7.6, we obtain

$$
\prod_{\epsilon \in \mathcal{S}_{1}} \frac{\epsilon \delta z_{0}}{\epsilon z_{0}} \equiv \prod_{\tilde{\sigma} e \equiv d_{1}} \tilde{\sigma}^{\mu_{\tilde{\sigma}}} \cdot \prod_{\tilde{\sigma} e \equiv d_{2}} \tilde{\sigma}^{-\mu_{\tilde{\sigma}}} \ldots \prod_{\tilde{\sigma} e \equiv d_{s}} \tilde{\sigma}^{-\mu_{\tilde{\sigma}}} \cdot\left(z^{M}\right) \quad\left(\bmod p^{n}\right)
$$

where

$$
M= \begin{cases}\left\langle\operatorname{path}\left(v_{0}, \delta v_{0}\right), \sum_{\sigma \in G_{n}} \mu_{\bar{\sigma}} \sigma e\right\rangle & \text { if } \iota=1 \\ \left\langle\operatorname{path}\left(v_{0}, \delta v_{0}\right), \sum_{\sigma \in G_{n}}\left(\mu_{\bar{\sigma}}-\mu_{\bar{\sigma}} w\right) \sigma e\right\rangle & \text { if } \iota=2 .\end{cases}
$$

By lemma 2.3, the duality $\langle$,$\rangle induces a pairing on H_{1}(\mathcal{G}, \mathbb{Z}) \times \mathcal{M}$. In the case $\iota=1$, one sees directly that $\sum_{\sigma \in G_{n}} \mu_{\bar{\sigma}} \sigma e$ has trivial image in $\mathcal{M}$, so that $M$ is zero. Consider now the case $\iota=2$. Since we are interested in computing $\underline{j}(\mathfrak{c})(\bar{\delta})$, we need only consider the image of the homomorphism $j(\mathfrak{c})$ in $\mathcal{N}_{\mathrm{sp}} \otimes \mathbb{Q}_{p}^{\times}$. Thus, we may view the above pairing as being defined on $H_{1}(\mathcal{G}, \mathbb{Z})^{-} \times \mathcal{M}_{\mathrm{sp}}$, where $H_{1}(\mathcal{G}, \mathbb{Z})^{-}$ indicates the "minus" eigenspace for the action of $w$ on $H_{1}(\mathcal{G}, \mathbb{Z})$, and we may assume from now on that $\operatorname{path}\left(v_{0}, \delta v_{0}\right)$ belongs to $H_{1}(\mathcal{G}, \mathbb{Z})^{-}$. One checks that the image $\iota \sum_{\sigma \in G_{n}} \mu_{\bar{\sigma}} \sigma e$ in $\mathcal{M}_{\mathrm{sp}}$ of the element $\sum_{\sigma \in G_{n}}\left(\mu_{\bar{\sigma}}-w \mu_{\bar{\sigma}}\right) \sigma e$ is trivial, so that also in this case $M$ is zero. Hence, in all cases

$$
\prod_{\epsilon \in \mathcal{S}_{1}} \frac{\epsilon \delta z_{0}}{\epsilon z_{0}} \equiv \prod_{\tilde{\sigma} e \equiv d_{1}} \tilde{\sigma}^{\mu_{\tilde{\sigma}}} \cdot \prod_{\tilde{\sigma} e \equiv d_{2}} \tilde{\sigma}^{-\mu_{\tilde{\sigma}}} \cdots \prod_{\tilde{\sigma} e \equiv d_{s}} \tilde{\sigma}^{-\mu_{\tilde{\sigma}}}\left(\bmod p^{n}\right) .
$$

Let $\operatorname{rec}_{p, n}: \tilde{G}_{\infty} \rightarrow G_{n}$ be the composite of the reciprocity map with the natural projection of $G_{\infty}$ onto $G_{n}$. Suppose that $\iota=1$. By lemma 7.6, the above relation yields the equality in $G_{n}$ :

$$
\operatorname{rec}_{p, n}\left(\prod_{\epsilon \in \mathcal{S}_{1}} \frac{\epsilon \delta z_{0}}{\epsilon z_{0}}\right)=\prod_{\sigma e \approx d_{1}} \sigma^{\omega_{d_{1}} \mu_{\bar{\sigma}}} \cdot \prod_{\sigma e \approx d_{2}} \sigma^{-\omega_{d_{2}} \mu_{\bar{\sigma}}} \cdots \prod_{\sigma e \approx d_{s}} \sigma^{-\omega_{d_{s}} \mu_{\bar{\sigma}}} .
$$


Recall the derivative $\mathcal{L}_{p, n}^{\prime}\left(\mathcal{N}_{\mathrm{sp}} / H, P_{0}\right) \in \mathcal{N}_{\mathrm{sp}} \otimes G_{n}$ defined in the formula (3) at the end of section 3. By the definition of the bijection $\kappa$ of lemma 2.2, the right hand side of the above equality can be written as

$$
\mathcal{L}_{p, n}^{\prime}\left(\mathcal{N}_{\mathrm{sp}} / H, P_{0}\right)(\bar{\delta})=\left\langle\operatorname{path}\left(v_{0}, \delta v_{0}\right), \sum_{g \in G_{n}} e_{n}(i)^{g} \otimes g^{-1}\right\rangle
$$

where, by an abuse of notation, $\sum_{g \in G_{n}} e_{n}(i)^{g} \otimes g^{-1}$ is viewed as an element of $\mathcal{M}_{\text {sp }} \otimes G_{n}$. When $\iota=2$, a similar computation shows that

$$
\iota \mathcal{L}_{p, n}^{\prime}\left(\mathcal{N}_{\mathrm{sp}} / H, P_{0}\right)(\bar{\delta})=\operatorname{rec}_{p, n}\left(\prod_{\epsilon \in \mathcal{S}_{1}} \frac{\epsilon \delta z_{0}}{\epsilon z_{0}}\right)
$$

By passing to the limit, one obtains in all cases

$$
\iota \mathcal{L}_{p}^{\prime}\left(\mathcal{N}_{\mathrm{sp}} / H, P_{0}\right)(\bar{\delta})=\operatorname{rec}_{p}\left(\prod_{\epsilon \in \mathcal{S}_{1}} \frac{\epsilon \delta z_{0}}{\epsilon z_{0}}\right)
$$

In other words, by definition of the map $\underline{j}$,

$$
\mathcal{L}_{p}^{\prime}\left(\mathcal{N}_{\mathrm{sp}} / H, P_{0}\right)^{\iota}=\underline{j}(\mathfrak{c})
$$

as was to be shown.

\section{References}

[BC] J-F. Boutot, H. Carayol, Uniformization p-adique des courbes de Shimura: les théorèmes de Cerednik et de Drinfeld, Astérisque 196-197 (1991) 45-158.

[BD1] M. Bertolini, H. Darmon, Heegner points on Mumford-Tate curves, Inv. Math. 126, Fasc. 3 (1996) 413-453.

[BD2] M. Bertolini, H. Darmon, Heegner points, $p$-adic L-functions and the CerednikDrinfeld uniformization, Inv. Math., to appear.

[Boi] X. Boichut, On the Mazur-Tate-Teitelbaum p-adic conjecture for elliptic curves with bad reduction at $p$, in preparation.

[BSDGP] K. Barré-Sirieix, G. Diaz, F. Gramain, G. Philibert, Une preuve de la conjecture de Mahler-Manin, Inv. Math. 124 (1996) 1-9.

[Cer] I.V. Cerednik, Uniformization of algebraic curves by discrete arithmetic subgroups of $\mathrm{PGL}_{2}\left(k_{w}\right)$ with compact quotient, (in Russian) Math. Sbornik 100 (1976) 59-88. Transl. in Math. USSR. Sb. 29 (1976) 55-78.

[Dag] H. Daghigh, McGill University PhD thesis, in progress.

[Dr] V.G. Drinfeld, Coverings of p-adic symmetric regions, Funct. Anal. Appl. 10 (1976) 29-40.

[Gr] B.H. Gross, Heights and special values of L-series, CMS Conference Proceedings, H. Kisilevsky, J. Labute, Eds., 7 (1987). 
[GS] R. Greenberg, G. Stevens, p-adic L-functions and p-adic periods of modular forms, Inv. Math. 111 (1993) 407-447.

[GVdP] L. Gerritzen, M. Van der Put, Schottky groups and Mumford curves, LNM 817, Springer, 1980.

[GZ] B.H. Gross, D. Zagier, Heegner points and derivatives of L-series, Inv. Math. 84 (1986) 225-320.

[Kl] C. Klingenberg, On p-adic L-functions on Mumford curves, In: p-adic Monodromy and the Birch and Swinnerton-Dyer Conjecture, B. Mazur and G. Stevens, eds., Contemporary Math. 165 (1991) 277-315.

[KKT] K. Kato, M. Kurihara, Tsuji, Forthcoming work.

[JL] H. Jacquet, R.P. Langlands, Automorphic forms on $\mathrm{GL}_{2}$, LNM 114, Springer.

[M] Y.I. Manin, p-adic automorphic functions, J. Sov. Math. 5 (1976) 279-333.

[MTT] B. Mazur, J. Tate, J. Teitelbaum, On p-adic analogues of the conjectures of Birch and Swinnerton-Dyer, Inv. Math. 84 (1986) 1-48.

[RT] K. Ribet, S. Takahashi, Parametrization of elliptic curves by Shimura curves and by classical modular curves, Proc. Nat. Acad. Sc. 94 (1997) 11110-11114.

[Sch] P. Schneider, Rigid-analytic L-transforms, In: Number Theory, Noordwijkerhout, LNM 1068, Springer, 1984.

[Sh] T. Shintani, On the construction of holomorphic cusp forms of half integral weight, Nagoya Math. J. 58 (1975) 83-126.

[Se] J-P. Serre, Arbres, amalgames, $S L_{2}$, Astérisque 46, Soc. Math. de France, 1977.

[T] J. Teitelbaum, Values of $p$-adic L-functions and a $p$-adic Poisson kernel, Inv. Math. 101 (1990) 395-410.

[Vi] M-F. Vigneras, Arithmétique des algèbres des quaternions, LNM 800, Springer. 